\title{
Improved Attenuation-Based Radar Precipitation Estimation Considering the Azimuthal Variabilities of Microphysical Properties
}

\author{
Hao Huang, ${ }^{\text {a,b }}$ Kun Zhao, ${ }^{\text {a,b }}$ HaOnan Chen,,${ }^{c, d}$ Dongming Hu, ${ }^{\text {e }}$ Peiling Fu, ${ }^{\text {f }}$ \\ QING LIN, ${ }^{\mathrm{e}}$ AND ZHENGWEI YANG ${ }^{\mathrm{a}, \mathrm{b}}$ \\ ${ }^{a}$ Key Laboratory for Mesoscale Severe Weather/MOE, and School of Atmospheric Sciences, Nanjing University, \\ Nanjing, Jiangsu, China \\ ${ }^{b}$ State Key Laboratory of Severe Weather, and Joint Center for Atmospheric Radar Research, \\ China Meteorological Administration, and Nanjing University, Beijing, China \\ ${ }^{c}$ Cooperative Institute for Research in the Atmosphere, Fort Collins, Colorado \\ ${ }^{d}$ NOAA/Earth System Research Laboratory, Boulder, Colorado \\ ${ }^{e}$ Guangdong Meteorological Observatory, Guangzhou, Guangdong, China \\ ${ }^{f}$ Guangzhou Meteorological Observatory, Guangzhou, Guangdong, China
}

(Manuscript received 9 November 2019, in final form 26 May 2020)

\begin{abstract}
The attenuation-based rainfall estimator is less sensitive to the variability of raindrop size distributions (DSDs) than conventional radar rainfall estimators. For the attenuation-based quantitative precipitation estimation (QPE), the key is to accurately estimate the horizontal specific attenuation $A_{H}$, which requires a good estimate of the ray-averaged ratio between $A_{H}$ and specific differential phase $K_{\mathrm{DP}}$, also known as the coefficient $\alpha$. In this study, a variational approach is proposed to optimize the coefficient $\alpha$ for better estimates of $A_{H}$ and rainfall. The performance of the variational approach is illustrated using observations from an S-band operational weather radar with rigorous quality control in south China, by comparing against the $\alpha$ optimization approach using a slope of differential reflectivity $Z_{\mathrm{DR}}$ dependence on horizontal reflectivity factor $Z_{H}$. Similar to the $Z_{\mathrm{DR}}$-slope approach, the variational approach can obtain the optimized $\alpha$ consistent with the DSD properties of precipitation on a sweep-to-sweep basis. The attenuation-based hourly rainfall estimates using the sweep-averaged $\alpha$ values from these two approaches show comparable accuracy when verified against the gauge measurements. One advantage of the variational approach is its feasibility to optimize $\alpha$ for each radar ray, which mitigates the impact of the azimuthal DSD variabilities on rainfall estimation. It is found that, based on the optimized $\alpha$ for radar rays, the hourly rainfall amounts derived from the variational approach are consistent with gauge measurements, showing lower bias $(1.0 \%)$, higher correlation coefficient $(0.92)$, and lower root-mean-square error $(2.35 \mathrm{~mm})$ than the results based on the sweepaveraged $\alpha$.
\end{abstract}

\section{Introduction}

Quantitative precipitation estimation (QPE) is one of the major missions in radar meteorology (Bringi and Chandrasekar 2001; Zhang et al. 2016). The uncertainty in the raindrop size distributions (DSDs) of precipitation and radar measurement errors are two major error sources for radar QPE (Lee 2006). For conventional weather radars without polarimetric capability, QPE is achieved by assuming power-law relations between rainfall rate $R$ and reflectivity factor $Z$, i.e., $Z-R$ relations (Marshall and Palmer 1948), which may suffer a lot from the DSD variabilities. After the polarimetric upgrade,

Corresponding author: Kun Zhao, zhaokun@nju.edu.cn weather radars can measure differential reflectivity factor $Z_{\mathrm{DR}}$ and specific differential phase shift $K_{\mathrm{DP}} /$ differential phase shift $\Phi_{\mathrm{DP}}$ in addition to the horizontal reflectivity factor $Z_{H}$. By including $Z_{\mathrm{DR}}$ and $K_{\mathrm{DP}}$ in the power-law estimators, e.g., $R\left(Z_{H}, Z_{\mathrm{DR}}\right), R\left(K_{\mathrm{DP}}\right)$, and $R\left(K_{\mathrm{DP}}, Z_{\mathrm{DR}}\right)$, the rainfall estimation will be less sensitive to the DSD uncertainty (Bringi et al. 2011; Chen et al. 2017; Ryzhkov et al. 2005a,b). By combining the polarimetric rainfall estimators and conventional $R(Z)$ estimators according to their error structures in terms of DSD variabilities and measurement errors, the QPE performance can be further improved (Ryzhkov et al. 2005a,b).

Recently, the relation between $R$ and specific attenuation $A$ is utilized for QPE (Diederich et al. 2015; Ryzhkov et al. 2014; Wang et al. 2014). As documented 
in Ryzhkov et al. (2014), the $R(A)$ estimator is less sensitive to DSD variabilities than $R(Z)$ and $R\left(K_{\mathrm{DP}}\right)$ estimators at $\mathrm{S}, \mathrm{C}$, and $\mathrm{X}$ bands. For attenuation-based QPE, the key factor is to estimate $A$ accurately. In their work, $A$ is estimated using the rain profiling algorithm called "ZPHI" from the attenuated $Z$ and the given total path integrated attenuation (PIA) (Testud et al. 2000). One of the ZPHI algorithm's advantages is its immunity to the miscalibration of $Z$. However, the accuracy of the $A$ estimated by the ZPHI algorithm heavily depends on the accuracy of the PIA. In the ZPHI algorithm, the PIA is calculated from $\Delta \Phi_{\mathrm{DP}}$ multiplied by a coefficient $\alpha$, where $\Delta \Phi_{\mathrm{DP}}$ is the change of differential phase shift over the propagation path and the coefficient $\alpha$ is the mean ratio of $A$ to $K_{\mathrm{DP}}$. In the $\mathrm{ZPHI}$ attenuation estimation algorithm (Ryzhkov et al. 2014; Testud et al. 2000), a fixed $\alpha$ is utilized. However, $\alpha$ is sensitive to the DSD variabilities and environmental temperature, which limits the accuracy of the ZPHI algorithm. The ZPHI algorithm is extended by adjusting $\alpha$ through minimizing the difference between the measured $\Phi_{\mathrm{DP}}$ and reconstructed $\Phi_{\mathrm{DP}}$ from retrieved $A$ (Bringi et al. 2001). However, since the coefficient $\alpha$ is sensitive to DSD variabilities, the constructed $\Phi_{\mathrm{DP}}$ may also lack precision (Gou et al. 2019; Gu et al. 2011; Ryzhkov et al. 2014). Gu et al. (2011) and Carey et al. (2000) tried to correct attenuation using the hot spot (HS) technique by adjusting the $\alpha$ values for the precipitation within the hot spot regions which usually have larger $Z_{\mathrm{DR}}$ values. This technique can mitigate the impact of DSD variabilities on attenuation correction but still suffer from the uncertainties to some degree. For example, the identification of the HS regions is not easy especially for radar rays with multiple HS regions. In addition, only two discrete $\alpha$ values are used for precipitation within and beyond the HS regions, but the actual $\alpha$ values usually have a higher spatial variance.

The DSD characteristics significantly vary for different rain types (e.g., tropical and continental rainfall). Thus, a slope of the $Z_{\mathrm{DR}}$ dependence on $Z_{H}$ in rain, which is an indicator of rain type, was used to optimize $\alpha$ on a sweep-to-sweep basis for attenuation-based QPE (Cocks et al. 2019; Ryzhkov and Zrnić 2019; Wang et al. 2019). This method is generally immune to the system biases in $Z_{H}$ and $Z_{\mathrm{DR}}$. And the optimized $\alpha$ values result in better rainfall estimates than a fixed $\alpha$ in their assessment (Cocks et al. 2019; Wang et al. 2019), making it promising for operational applications. Nevertheless, limitations exist in this method too, according to Wang et al. (2019). First, this method derives a single $\alpha$ value for a whole radar sweep. However, the DSD properties, especially the mean raindrop size, vary in different regions of a radar sweep. Using a single $\alpha$ value may introduce underestimation (overestimation) of rainfall for the echoes with small (large) mean raindrop size. On the other hand, to optimize an $\alpha$ value from the $Z_{\mathrm{DR}}$ slope, this method requires a large number of valid rainfall samples; the threshold is 30000 in Wang et al. (2019). If a radar sweep does not have a sufficient number of valid samples, a default or recently updated $\alpha$ value is used until a sufficient number of samples is accumulated. This strategy mitigates the impact of measurement errors and the skewed distribution of the $Z_{H}-Z_{\mathrm{DR}}$ pairs; however, this may enlarge the impact of the DSD variability for the sweeps without enough samples.

Considering the potential differences in DSD characteristics in different regions of a radar sweep, one may improve the performance of attenuation-based QPE if a radar sweep is segmented into subregions with different $\alpha$ values used for them. In our paper, this possibility is investigated using a variational algorithm. In this algorithm, the propagation effect (attenuation and phase shift) is considered in the forward operator and optimized when minimizing a cost function; the horizontal specific attenuation $A_{H}$, differential attenuation $A_{\mathrm{DP}}$, and $K_{\mathrm{DP}}$ are parameterized using $Z_{H}$ and $Z_{\mathrm{DR}}$. After estimating attenuation using the variational approach, the optimized coefficient $\alpha$ are derived for QPE based on $R(A)$ estimators, in which the azimuthal variabilities of $\alpha$ are considered. The paper is organized as follows. The dataset used for this study and the relevant approaches for attenuation estimation and QPE are introduced in section 2. In section 3, the measurements of an operational radar and surrounding gauges in south China are used to evaluate the proposed algorithm. Conclusions and discussions are given in section 4 .

\section{Data and methods}

\section{a. Dataset}

Influenced by the East Asian summer monsoon, heavy rainfall usually occurs in south China from May to June, causing enormous calamity (Luo et al. 2017). Since 2016, the Chinese operational radar network in south China has been gradually upgraded with polarimetric capability. As part of the operational radar network, the S-band polarimetric radar in Guangzhou City, i.e., GZ SPOL, has been proved to have good performance and flexibility for scientific studies (Huang et al. 2018; Wu et al. 2018). Therefore, GZ SPOL is used in this paper as the prototype for algorithm validation. Accordingly, all the retrieval models in the paper are constructed based on the DSD data collected from a two-dimensional video disdrometer (2DVD) in south China in May-July in 2016 and 2017. The location of GZSPOL and the 2DVD can 
be found in Fig. 1 of Huang et al. (2018). In the construction of the algorithms, polarimetric variables and $R$ are calculated from the DSD data according to formulas in Cao et al. (2012), with the scattering amplitudes estimated using the T-matrix method (Mishchenko et al. 2000). The axis ratios and terminal velocities of raindrops are parameterized from raindrop diameters following Brandes et al. (2002). In the QPE evaluation, the algorithms are mainly applied to the GZ SPOL measurements in precipitation events during May and June 2016; the radar scans at $1.5^{\circ}$ elevation angle are used. These radar-derived rainfall rates are temporally integrated and compared against the accumulated rainfall measured by gauges within the distance of $75 \mathrm{~km}$ from the radar, in which the gauge data are quality controlled using a speckle filter described in Huang et al. (2018).

In the variational approach, the quality of polarimetric radar data is crucial for its success. The quality control procedures are similar to those in Huang et al. (2018) and are listed below. For GZ SPOL, the reflectivity is calibrated by an internal system using generated test signals and the system is annually maintained, which guarantees the $Z_{H}$ precision to be within $1 \mathrm{~dB}$ (Chen et al. 2014). Then, to mitigate the impact of the nonmeteorological echoes on the retrieval, the data with the copolar correlation coefficient $\rho_{\mathrm{hv}}$ lower than 0.85 are removed. The remaining echoes having less than 20 consecutive range gates are also removed. The differential reflectivity $Z_{\mathrm{DR}}$ is calibrated by monitoring the echo with $Z_{H}$ between 10 and $20 \mathrm{~dB} Z$, for which the averaged intrinsic $Z_{\mathrm{DR}}$ value is $0.14 \mathrm{~dB}$ according to DSD statistics. In the processing of $\Phi_{\mathrm{DP}}$, the system bias is estimated from the statistics of the $\Phi_{\mathrm{DP}}$ values of the meteorological echo close to the radar site using the method in Maesaka et al. (2012). Then, the $\Phi_{\mathrm{DP}}$ measurements with the differences from the values at the neighboring range gates greater than $35^{\circ}$ are removed and refilled by linear interpolation after unfolding. After the quality control, the random errors of $Z_{H}$, $Z_{\mathrm{DR}}$, and $\Phi_{\mathrm{DP}}$ are assumed to be $1 \mathrm{~dB} Z, 0.2 \mathrm{~dB}$, and $2.0^{\circ}$, respectively, according to the specifications of GZ SPOL (Huang et al. 2018).

\section{b. Review of attenuation-based $Q P E$}

In Ryzhkov et al. (2014), attenuation is estimated using the ZPHI algorithm before QPE. The ZPHI algorithm was originally designed for spaceborne rain radar of the Tropical Rainfall Measuring Mission (TRMM). It was then applied to ground-based polarimetric radars by Testud et al. (2000) and has been utilized in many studies (Bringi et al. 2001; Gou et al. 2019; Gu et al. 2011; Ryzhkov et al. 2014). To derive the ZPHI algorithm, $A_{H}$ is assumed to be related to the horizontal reflectivity factor by

$$
A_{H}(r)=a(r)\left[Z_{h}(r)\right]^{b},
$$

where $Z_{h}$ is the horizontal reflectivity factor $\left(\mathrm{mm}^{6} \mathrm{~m}^{-3}\right)$. Parameter $b$ is nearly constant for GZ SPOL $(b=0.65)$ according to the scattering simulation using the DSD data. With $a(r)$ assumed to be constant along each radar beam, the $A_{H}$ profile for the path between $r_{0}$ and $r_{1}$ can be estimated using the measured (i.e., attenuated) reflectivity factor $Z_{a}$ and the corresponding total PIA, following

$$
A_{H}(r)=\frac{\left[Z_{a}(r)\right]^{b}\left(e^{0.23 b \mathrm{PIA}}-1\right)}{I\left(r_{0}, r_{1}\right)+I\left(r, r_{1}\right)\left(e^{0.23 b \mathrm{PIA}}-1\right)},
$$

where

$$
I\left(r_{0}, r_{1}\right)=0.46 b \int_{r_{0}}^{r_{1}}\left[Z_{a}(s)\right]^{b} d s,
$$

and

$$
I\left(r, r_{1}\right)=0.46 b \int_{r}^{r_{1}}\left[Z_{a}(s)\right]^{b} d s .
$$

Note that the uncertainty on attenuation estimation and QPE caused by the assumption of constant $a(r)$ in (1) is less than $20 \%$ as shown in Ryzhkov et al. (2014). In (2), the only unknown for the $A_{H}$ estimation is the PIA between path $\left(r_{0}, r_{1}\right)$. For polarimetric radars, PIA is usually calculated from path integrated differential phase $\left(\Delta \Phi_{\mathrm{DP}}\right)$ over the path $\left(r_{0}, r_{1}\right)$ (Bringi et al. 2001; Ryzhkov et al. 2014; Testud et al. 2000) by

$$
\operatorname{PIA}\left(r_{0}, r_{1}\right)=\alpha\left[\Phi_{\mathrm{DP}}\left(r_{1}\right)-\Phi_{\mathrm{DP}}\left(r_{0}\right)\right]=\alpha \Delta \Phi_{\mathrm{DP}},
$$

where $\alpha$ is the ray-averaged ratio between $A_{H}$ and $K_{\mathrm{DP}}$.

After estimating $A_{H}$, the rainfall rate can be calculated from $R\left(A_{H}\right)$ estimators constructed for GZ SPOL. As documented in Ryzhkov et al. (2014), the relation between $R$ and $A_{H}$ is less sensitive to DSD variabilities than $R\left(Z_{H}\right)$ and $R\left(K_{\mathrm{DP}}\right)$ especially for $S$ band, but it is sensitive to radar wavelength and temperature. To minimize the impact of these two factors, the $R\left(A_{H}\right)$ estimators are fitted at $0^{\circ}-30^{\circ} \mathrm{C}$ with a $1^{\circ} \mathrm{C}$ interval for GZ SPOL using a nonlinear least squares approach. Taking the regression result at $20^{\circ} \mathrm{C}\left(R=2311.7 A_{H}^{0.95}\right)$ as an example (Fig. 1), $R$ can be well parameterized by $A_{H}$. The correlation coefficient (CC) between the $R$ values calculated from DSDs and those predicted from $R\left(A_{H}\right)$ is close to 1 and the root-mean-square error (RMSE) is low $\left(1.99 \mathrm{~mm} \mathrm{~h}^{-1}\right)$. The definition of CC, RMSE, 


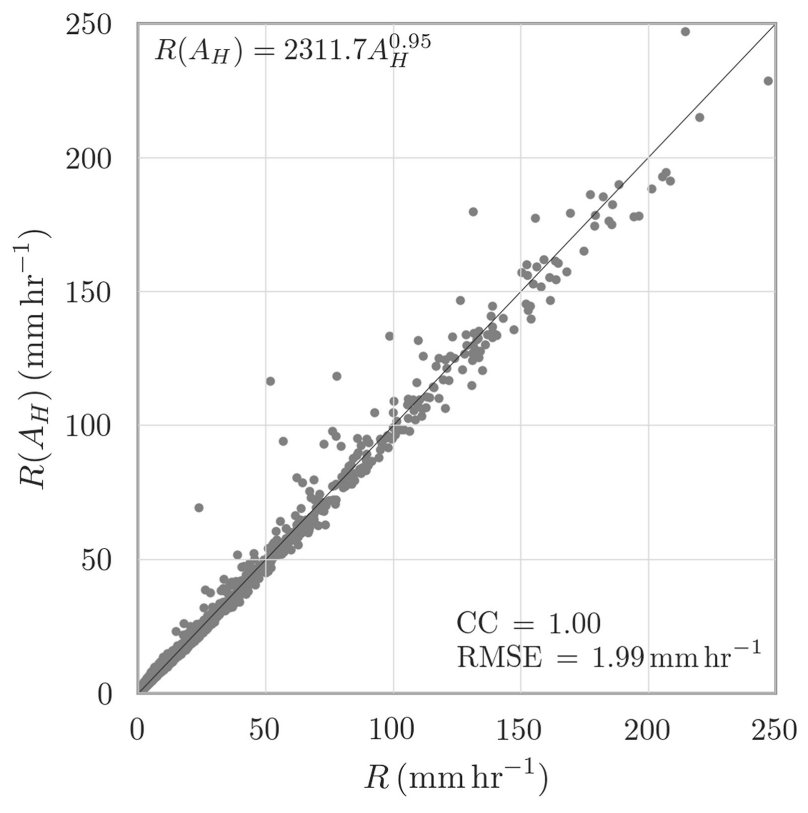

FIG. 1. Scatterplot of $R$ directly calculated from the DSDs vs those calculated from $R\left(A_{H}\right)$ at $20^{\circ} \mathrm{C}$ for GZ SPOL simulated from the measurements of the 2DVD.

normalized error (NE), and relative bias ( $\mathrm{RB}$ ) can be found in Huang et al. $(2018,2020)$. The dependence of $R\left(A_{H}\right)$ on temperature is also shown in Table 1 . When temperature changes from $0^{\circ}$ to $30^{\circ} \mathrm{C}$, the exponent keeps nearly constant $(0.95)$ and the intercept raises from 1361 to 2923. In practical applications, the $R\left(A_{H}\right)$ estimator for a specific radar range gate is selected from prefitted ones according to the temperature from the interpolation of the sounding measurements. The sounding station at Qingyuan (station number 59280) is within the coverage of GZ SPOL, and it provides measurements of temperature profiles twice a day.

The key for the attenuation-based QPE is the accuracy of the $\alpha$ used in (5). In the initial implementation of the ZPHI algorithm (Testud et al. 2000) and the attenuation-based QPE (Ryzhkov et al. 2014), a fixed $\alpha$ is used. However, as revealed by the simulation using DSD data (Fig. 2), the ratio of $A_{H}$ to $K_{\mathrm{DP}}(\alpha)$ changes in terms of mean raindrop size (denoted by mass-weighted mean diameter, $\left.D_{m}\right)$ as well as temperature. For a specific

TABLE 1 . The $R\left(A_{H}\right)$ estimators and $\alpha$ values at different temperatures $\left(0^{\circ}, 10^{\circ}, 20^{\circ}\right.$, and $\left.30^{\circ} \mathrm{C}\right)$ for GZ SPOL.

\begin{tabular}{ccc}
\hline \hline Temperature $\left({ }^{\circ} \mathrm{C}\right)$ & $R\left(A_{H}\right)$ & $\alpha=A_{H} / K_{\mathrm{DP}}$ \\
\hline 0 & $R=1361.3 A_{H}^{0.95}$ & 0.036 \\
10 & $R=1789.5 A_{H}^{0.95}$ & 0.027 \\
20 & $R=2311.7 A_{H}^{0.95}$ & 0.021 \\
30 & $R=2922.8 A_{H}^{0.95}$ & 0.016 \\
\hline
\end{tabular}

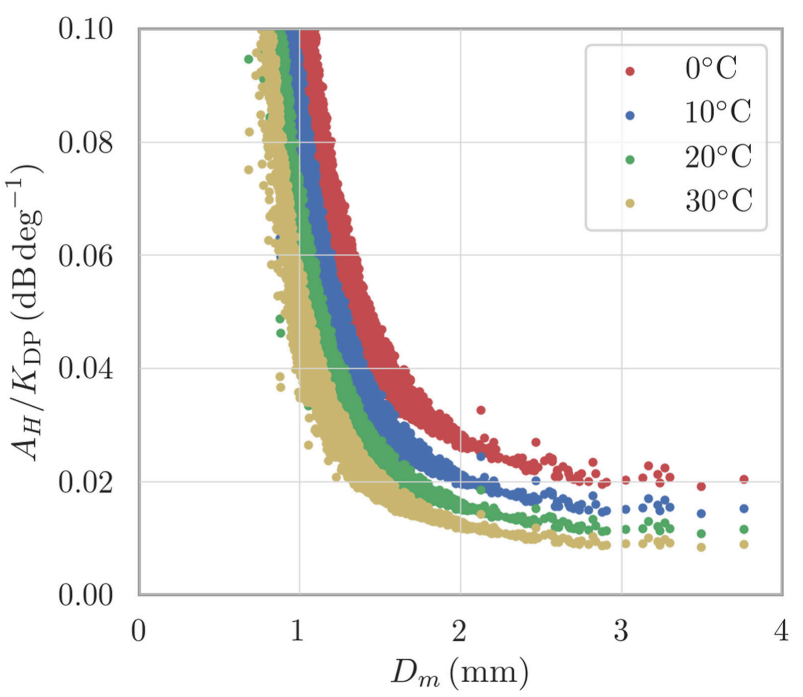

FIG. 2. Scatterplot of the mass-weighted mean diameter $D_{m}$ vs $A_{H} / K_{\mathrm{DP}}$ at $0^{\circ} \mathrm{C}$ (red), $10^{\circ} \mathrm{C}$ (blue), $20^{\circ} \mathrm{C}$ (green), and $30^{\circ} \mathrm{C}$ (yellow) for GZ SPOL simulated from the measurements of the 2DVD.

temperature, $\alpha$ generally decreases when $D_{m}$ increases (Ryzhkov et al. 2014). As a result, the mean $\alpha$ can vary sweep by sweep or even ray by ray due to the spatial variation of precipitation. Two methods, i.e., the method based on the $Z_{\mathrm{DR}}$ slope and the variational approach, for $\alpha$ optimization are introduced below.

\section{c. Estimating $\alpha$ using $Z_{D R}$ slope}

As DSD properties can be indicated by the rainfall type, which can be distinguished from the slope of $Z_{\mathrm{DR}}$ dependence on $Z_{H}$, Wang et al. (2019) proposed to optimize $\alpha$ from a $Z_{\mathrm{DR}}$ slope on a sweep-to-sweep basis. For a radar sweep, a $Z_{\mathrm{DR}}$ slope $K$ is calculated from the $Z_{H}$ and $Z_{\mathrm{DR}}$ measurements. Then, a relation between $\alpha$ and $K$, which is constructed from DSD simulations, is adopted to calculate the $\alpha$ for the sweep. In our paper, for the verification of the variational approach detailed in the next subsection, we use the QPE results based on the $Z_{\mathrm{DR}}$ slope method similar to that in Wang et al. (2019) as a benchmark.

In Wang et al. (2019), the bilinear $\alpha-K$ relation is obtained from the DSD measurements in Oklahoma. The DSD data with simulated $Z_{H}$ values between 20 and $50 \mathrm{~dB} Z$ are separated into bins with $2-\mathrm{dB} Z$ width. Within each bin, the simulated $Z_{\mathrm{DR}}$ values are sorted and DSD data are separated into five groups with equal sizes according to the sorted $Z_{\mathrm{DR}}$ values. For each group, the $\alpha$ value is calculated by $\sum A_{H} / \sum K_{\mathrm{DP}}$, where summation is performed over all DSDs; the $K$ value is the difference of the median $Z_{\mathrm{DR}}$ values for two $Z_{H}$ bins centered at 20 and $50 \mathrm{~dB} Z$ divided by 30 . Then, the bilinear $\alpha-K$ relation is obtained from the five $\alpha-K$ pairs. 
In our paper, this method is applied to DSD measurements in south China, as shown in Fig. 3. Since the rainfall in Guangzhou is more tropical than in Oklahoma, the $\alpha-K$ pairs in Fig. 3 indicates that a larger $\alpha$ exists for a specific $K$ than that in Oklahoma. It is also found that five points (Fig. 3) are not enough for a robust relation fitting. Thus, we manually adjust the relation from Oklahoma (the red dashed line) for south China (the blue dashed line), as follows:

$$
\begin{array}{ll}
\alpha=0.055-0.75 K, & \text { if } \quad K<0.0467, \\
\alpha=0.02, & \text { if } \quad K \geq 0.0467 .
\end{array}
$$

With (6), we can now determine the $\alpha$ from the $Z_{H^{-}}$ $Z_{\mathrm{DR}}$ pairs, for which the radar sweeps at $1.5^{\circ}$ elevation are used. The $Z_{H}-Z_{\mathrm{DR}}$ pairs are selected by the criteria: 1) data below $4 \mathrm{~km}$ to mitigate potential contamination from ice particles; 2) $\rho_{\mathrm{hv}}>0.98$; 3) $-4<Z_{\mathrm{DR}}<4 \mathrm{~dB}$; and 4) $20<Z_{H}<50 \mathrm{~dB} Z$. The $Z_{H}-Z_{\mathrm{DR}}$ pairs are separated into $16 Z_{H}$ bins with 2 -dBZ width from 20 to $50 \mathrm{~dB} Z$; within each bin, a median $Z_{\mathrm{DR}}$ value is calculated if the sample size within the bin is larger than 200 . Then, the $Z_{\mathrm{DR}}$ slope $K$ is obtained from the linear regression fitting of the median $Z_{\mathrm{DR}}$ values in terms of the $Z_{H}$ bin centers. Afterward, the optimized $\alpha$ is calculated from $K$ using the bilinear $\alpha-K$ relation, i.e., (6). Note that if the sample size of the valid $Z_{H}-Z_{\mathrm{DR}}$ pairs in a radar sweep is smaller than 30000 , the recently updated $\alpha$ is used until enough samples are accumulated.

\section{$d$. Estimating $\alpha$ using a variational approach}

To get the $\alpha$ values on a ray-to-ray basis, a variational approach is proposed in this paper. In the variational approach, the forward operators are based on the relations among the polarimetric variables. According to Gorgucci et al. (1992), $Z_{H}(\mathrm{~dB} Z), Z_{\mathrm{DR}}(\mathrm{dB})$, and $K_{\mathrm{DP}}\left({ }^{\circ} \mathrm{km}^{-1}\right)$ triplets reside within a limited three-dimensional space for rainfall, and $K_{\mathrm{DP}}$ can be well related to $Z_{H}$ and $Z_{\mathrm{DR}}$, known as a self-consistent relation $K_{\mathrm{DP}}\left(Z_{H}, Z_{\mathrm{DR}}\right)$. Similarly, $A_{H}\left(\mathrm{~dB} \mathrm{~km}^{-1}\right)$ and $A_{\mathrm{DP}}\left(\mathrm{dB} \mathrm{km}^{-1}\right)$ can also be parameterized using $Z_{H}$ and $Z_{\mathrm{DR}}$, i.e., $A_{H}\left(Z_{H}, Z_{\mathrm{DR}}\right)$ and $A_{\mathrm{DP}}\left(Z_{H}, Z_{\mathrm{DR}}\right)$. These relations can be sensitive to temperature. Thus, we fit these three relations at $0^{\circ}-30^{\circ} \mathrm{C}$ with a $1^{\circ} \mathrm{C}$ interval using the 2DVD data for GZ SPOL. To parameterize $K_{\mathrm{DP}}, A_{H}$, and $A_{\mathrm{DP}}$ from $Z_{H}$ and $Z_{\mathrm{DR}}$, the relations are also selected from the prefitted ones according to the temperature from the sounding measurements. The examples of fitted $K_{\mathrm{DP}}\left(Z_{H}, Z_{\mathrm{DR}}\right), A_{H}\left(Z_{H}, Z_{\mathrm{DR}}\right)$, and $A_{\mathrm{DP}}\left(Z_{H}, Z_{\mathrm{DR}}\right)$ at $20^{\circ} \mathrm{C}$ are shown in Fig. 4 , which can be formulated as

$$
\begin{aligned}
K_{\mathrm{DP}}= & -3.52 \times 10^{-7} \times Z_{h}^{1.00} \\
& \times\left(Z_{\mathrm{DR}}^{3}-10.7 Z_{\mathrm{DR}}^{2}+45.1 Z_{\mathrm{DR}}-90.4\right),
\end{aligned}
$$

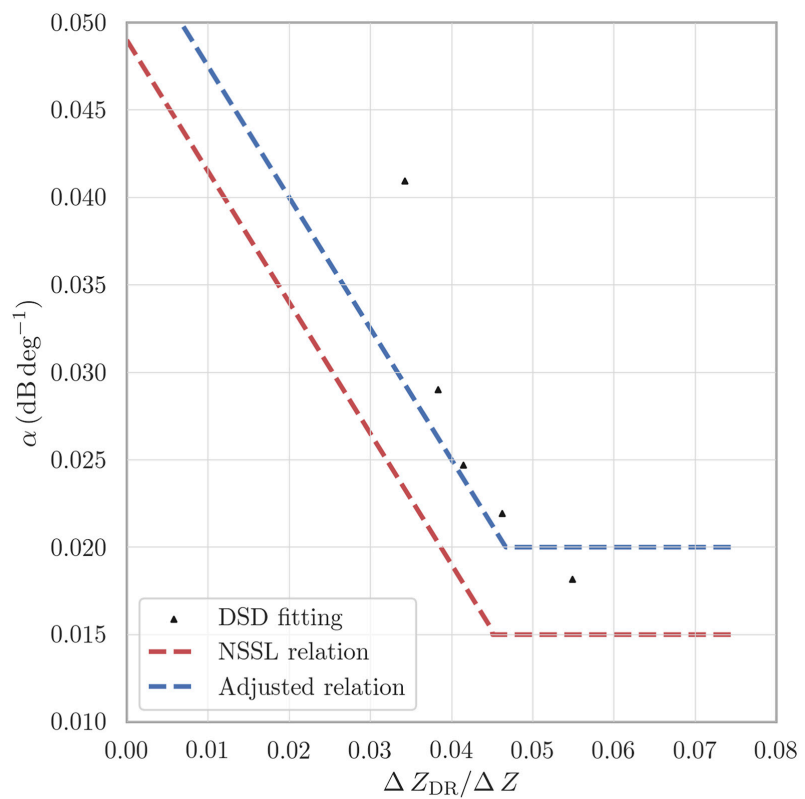

FIG. 3. The relation between factor $\alpha$ (dB per degree) and $Z_{\mathrm{DR}}$ slope as simulated from DSD observations collected by a 2DVD in south China. Black triangles represent each simulated variable value for the $0 \%-20 \%, 20 \%-40 \%, 40 \%-60 \%, 60 \%-80 \%$, $80 \%-100 \%$ percentile groups of $Z_{\mathrm{DR}}$. The red dashed line represents the bilinear fits from the DSDs in Oklahoma (Wang et al. 2019). The blue dashed line is the adjusted relation for south China.

$$
\begin{aligned}
A_{H}= & -2.52 \times 10^{-8} \times Z_{h}^{1.07} \\
& \times\left(Z_{\mathrm{DR}}^{3}-8.9 Z_{\mathrm{DR}}^{2}+26.7 Z_{\mathrm{DR}}-30.0\right), \\
A_{\mathrm{DP}}= & 1.03 \times 10^{-10} \times Z_{h}^{0.99} \\
& \times\left(Z_{\mathrm{DR}}^{3}+36.2 Z_{\mathrm{DR}}^{2}-183.9 Z_{\mathrm{DR}}+616.6\right),
\end{aligned}
$$

where $Z_{h}\left(\mathrm{~mm}^{6} \mathrm{~m}^{-3}\right)$ equals to $10^{0.1 Z_{H}}$. It can be found all the $K_{\mathrm{DP}}, A_{H}$, and $A_{\mathrm{DP}}$ can be well parameterized by $Z_{H}$ and $Z_{\mathrm{DR}}$, with most of the samples located near the one-to-one lines, which means these relations are not very sensitive to DSD variabilities.

With the above relations, the attenuation of a radar sweep can be estimated ray by ray. We first assume a state vector $\mathbf{x}$ containing the unattenuated $Z_{H}$ and $Z_{\mathrm{DR}}$ for the $n$-gate radar ray,

$$
\begin{aligned}
\mathbf{x}= & {\left[Z_{H}(1), Z_{H}(2), \cdots, Z_{H}(n), Z_{\mathrm{DR}}(1),\right.} \\
& \left.\times Z_{\mathrm{DR}}(2), \cdots, Z_{\mathrm{DR}}(n)\right]^{\mathrm{T}} .
\end{aligned}
$$

The measurement vector $\mathbf{y}$ containing the measured reflectivity $Z_{H}^{m}$, measured differential reflectivity $Z_{\mathrm{DR}}^{m}$, and measured differential phase shift $\Phi_{\mathrm{DP}}^{m}$ at each ray can be expressed as 

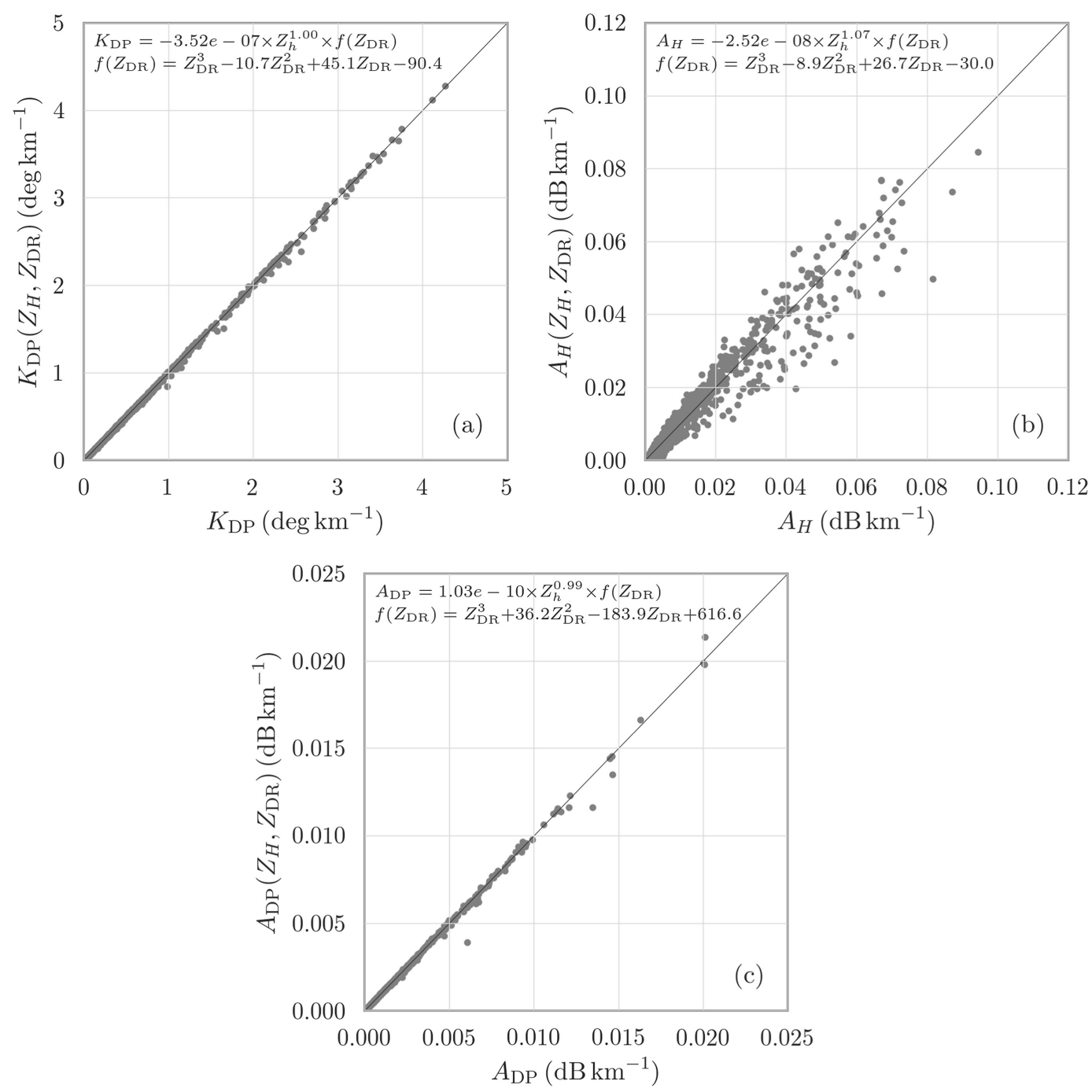

FIG. 4. Scatterplots of the regression results of the parameterizations of (a) $K_{\mathrm{DP}}$, (b) $A_{H}$, and (c) $A_{\mathrm{DP}}$ in terms of $Z_{H}$ and $Z_{\mathrm{DR}}$ at $20^{\circ} \mathrm{C}$ for GZ SPOL simulated from the measurements of the 2DVD. The text at the left top corner of the subplots indicates the fitted relations.

$$
\begin{aligned}
\mathbf{y}_{Z_{H}} & =\left[Z_{H}^{m}(1), Z_{H}^{m}(2), \cdots, Z_{H}^{m}(n)\right]^{\mathrm{T}}, \\
\mathbf{y}_{Z_{\mathrm{DR}}} & =\left[Z_{\mathrm{DR}}^{m}(1), Z_{\mathrm{DR}}^{m}(2), \cdots, Z_{\mathrm{DR}}^{m}(n)\right]^{\mathrm{T}}, \\
\mathbf{y}_{\Phi_{\mathrm{DP}}} & =\left[\Phi_{\mathrm{DP}}^{m}(1), \Phi_{\mathrm{DP}}^{m}(2), \cdots, \Phi_{\mathrm{DP}}^{m}(n)\right]^{\mathrm{T}}, \\
\mathbf{y} & =\left[\mathbf{y}_{Z_{H}} ; \mathbf{y}_{Z_{\mathrm{DR}}} ; \mathbf{y}_{\Phi_{\mathrm{DP}}}\right] .
\end{aligned}
$$

Since $K_{\mathrm{DP}}, A_{H}$, and $A_{\mathrm{DP}}$ can be parameterized from the unattenuated $Z_{H}$ and $Z_{\mathrm{DR}}$ using (7)-(9), the polarimetric measurements can be predicted from $\mathbf{x}$ with the propagation effects (attenuation and phase accumulation) considered in the integration terms. This is known as the forward operator $H(\mathbf{x})$. Then, the difference between the predicted and measured polarimetric measurements normalized by the error covariance matrix $\mathbf{R}$ is known as the cost function $J$,

$$
\begin{aligned}
J & =J_{b}+J_{y}, \\
J_{b}(\mathbf{x}) & =\frac{1}{2}\left(\mathbf{x}-\mathbf{x}_{b}\right)^{\mathrm{T}} \mathbf{B}^{-1}\left(\mathbf{x}-\mathbf{x}_{b}\right), \\
J_{y}(\mathbf{x}) & =\frac{1}{2}[H(\mathbf{x})-\mathbf{y}]^{\mathrm{T}} \mathbf{R}^{-1}[H(\mathbf{x})-\mathbf{y}] .
\end{aligned}
$$

As the conventional cost function used in data assimilation, $\mathbf{x}_{b}$ and $\mathbf{B}$ are the a priori and its error covariance matrix, and $J_{b}$ is the corresponding cost function. In this study, we do not use any a priori constraint for $\mathbf{x}$ and $J_{b}$ is set to 0 hereafter. In the error covariance matrix $\mathbf{R}$ for $\mathbf{y}$, we assume the errors of $Z_{H}, Z_{\mathrm{DR}}$, and $\Phi_{\mathrm{DP}}$ are generally 
independent from each other and are independent from gate to gate according to Hogan (2007) and Cao et al. (2013). Thus, $\mathbf{R}$ is composed of diagonal matrices $\mathbf{R}_{Z_{H}}$, $\mathbf{R}_{Z_{\mathrm{DR}}}$, and $\mathbf{R}_{\Phi_{\mathrm{DP}}}$ :

$$
\mathbf{R}=\left[\begin{array}{ccc}
\mathbf{R}_{Z_{H}} & & \mathbf{0} \\
& \mathbf{R}_{Z_{\mathrm{DR}}} & \\
\mathbf{0} & & \mathbf{R}_{\Phi_{\mathrm{DP}}}
\end{array}\right]
$$

with $\mathbf{O}$ representing the zero matrices.

To obtain the optimized polarimetric radar data, the key process is to minimize the cost function $J$ which contains the nonlinear forward operator $H(\mathbf{x})$, in which the limited-memory Broyden-Fletcher-Goldfarb-Shanno algorithm for bound constrained large-scale nonlinear optimization (LBFGS-B) is utilized (Byrd et al. 1995). Since the raindrops are statistically oblate (Brandes et al. 2002), nonnegative constraints are used for unattenuated $Z_{\mathrm{DR}}$ in the optimization. The minimization of a radar ray starts from a first guess, we use $30 \mathrm{~dB} Z$ and $0.5 \mathrm{~dB}$ for all the $Z_{H}$ and $Z_{\mathrm{DR}}$ of the ray. In later iterations of the minimization, the value of the cost function and its gradient in terms of $\mathbf{x}$ is required. The gradient term with the a priori term omitted can be written as

$$
\mathbf{g}(\mathbf{x})=\mathbf{H}^{\mathrm{T}} \mathbf{R}^{-1}[H(\mathbf{x})-\mathbf{y}],
$$

where $\mathbf{H}$ is the Jacobian matrix of the forward operator. In the minimization, it is obtained using automatic differentiation (Hogan 2014).

To further increase the accuracy of the result, the continuity of the precipitation is further introduced to the variational analysis by using radial $B$-spline filters in a similar way to Huang et al. (2018). The $B$-spline filter can enforce the smoothness of the state variables, which could mitigate the impact of random measurement errors on the state variables. The formulas are similar to those used in Huang et al. (2018) and not repeated here.

After the minimization of the cost function, the unattenuated $Z_{H}$ and $Z_{\mathrm{DR}}$ in the state vector are considered to be optimized for the ray. Then, the calculation is continued for the next radar ray in the same sweep until finished. Afterward, $A_{H}$ and $K_{\mathrm{DP}}$ can be calculated from the optimized state variables using (7) and (8). Then, the $\alpha$ is estimated for each radar ray by $\sum A_{H} / \sum K_{\mathrm{DP}}$ where summation is performed for all valid gates of the ray. In addition, to better compare against the method based on the $Z_{\mathrm{DR}}$ slope, we further calculate a single $\alpha$ for the whole sweep by $\sum A_{H} / \sum K_{\mathrm{DP}}$ where summation is performed for all valid gates of the sweep.

\section{e. Different configurations for attenuation-based $Q P E$}

As documented in section $2 \mathrm{~b}$, the accuracy of attenuationbased rainfall estimation is closely related to the accuracy of $\alpha$ used for $A_{H}$ estimation. Six different configurations for PIA calculation in (5) and the $R(A)$ estimation are considered for further comparison. The first one is to use $\alpha$ values fitted from the DSD measurements $\left(A_{H}=\right.$ $\left.\alpha K_{\mathrm{DP}}\right)$. To consider the sensitivity of $\alpha$ to temperature, the $\alpha$ values are fitted at $0^{\circ}-30^{\circ} \mathrm{C}$ with a $1^{\circ} \mathrm{C}$ interval, with the examples shown in Table 1 . In this configuration (denoted as CONV), the coefficient $\alpha$ in (5) is chosen from the fitted values according to the mean temperature of the radar sweep. In (5), another important issue is the way to determine $\Delta \Phi_{\mathrm{DP}}$ over the path $\left(r_{0}, r_{1}\right)$. For CONV, the $\Phi_{\mathrm{DP}}$ measurements are filtered by a ninegate running mean filter and the values of $\Phi_{\mathrm{DP}}\left(r_{0}\right)$ and $\Phi_{\mathrm{DP}}\left(r_{1}\right)$ are the median of the $\Phi_{\mathrm{DP}}$ values at the seven gates near the gates $r_{0}$ and $r_{1}$. The second configuration (denoted by SLP) uses the same way to determine $\Delta \Phi_{\mathrm{DP}}$ as for CONV, and its $\alpha$ is determined from the $Z_{\mathrm{DR}}$ slope based on the $\alpha-K$ relation adjusted for south China, i.e., (6).

The other four configurations involve the results from the variational analysis described in section $2 \mathrm{~d}$. In these four configurations, $\Delta \Phi_{\mathrm{DP}}$ is obtained from the difference of $\phi_{\mathrm{DP}}$ between the gates $r_{0}$ and $r_{1}$, in which $\phi_{\mathrm{DP}}$ is predicted from the optimized state variable of the variational retrieval. After the optimization, the $\phi_{\mathrm{DP}}$ is close to $\Phi_{\mathrm{DP}}$ but with the random errors filtered out. In such a way, the $\Delta \Phi_{\mathrm{DP}}$ is expected to result in better attenuation and rainfall rate than the one used in CONV and SLP. For the selection of $\alpha$, the third (fourth) configurations, denoted as CP (SP), uses the same strategy as in the CONV (SLP). For the fifth configuration, we use the sweepaveraged $\alpha$ calculated from the variational approach. This configuration is denoted as the VU (the variational approach with a uniform $\alpha$ ). For the last configuration, the $\alpha$ estimated for each ray by the variational approach is used. In such a way, the impact of DSD variabilities on $\alpha$ is considered ray by ray, which is denoted as the VAZ (the variational approach with $\alpha$ values optimized for azimuths).

\section{QPE evaluation}

\section{a. An example of attenuation estimation}

On 28 May 2016, a mesoscale convective system passed over GZ SPOL. The plan position indicator (PPI) images of the radar scan at $1.5^{\circ}$ elevation at 0600 UTC are shown in Fig. 5. The measured $Z_{H}, Z_{\mathrm{DR}}$, and $\Phi_{\mathrm{DP}}$ are exhibited 

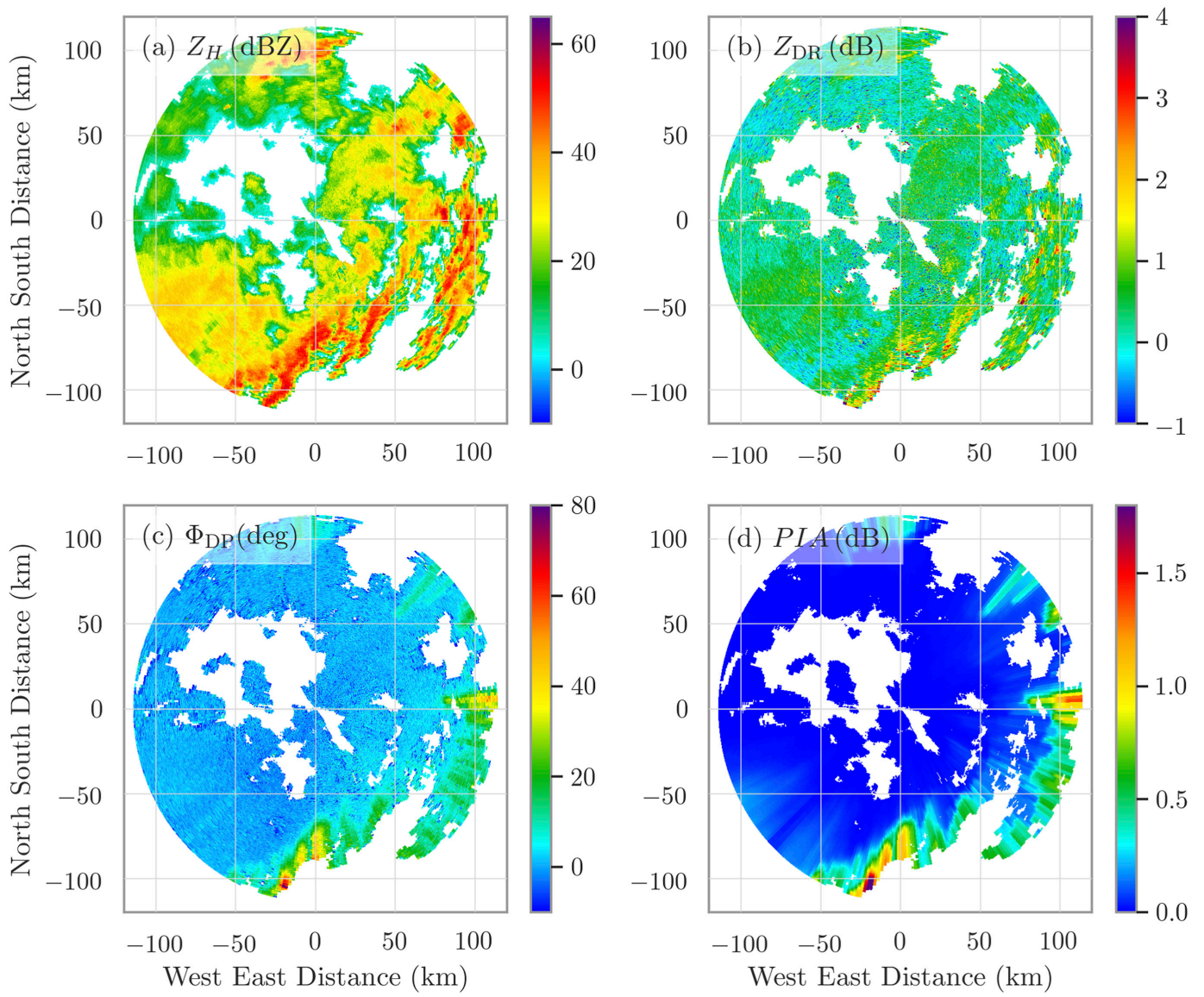

FIG. 5. The PPI images of a precipitation event collected by GZ SPOL at 0600 UTC 28 May 2016 at the elevation angle of $1.5^{\circ}$. (a)-(c) The radar measurements of $Z_{H}, Z_{\mathrm{DR}}$, and $\Phi_{\mathrm{DP}}$, respectively; (d) the PIA calculated from the optimized state variables of the variational analysis.

by Figs. 5a-c. Multiple quasi-linear convections are distributed to the southeast of the radar site, and large areas of stratiform precipitation exist to the northwest of the convections. In the stratiform regions, the $Z_{\mathrm{DR}}$ values are generally low and the $\Phi_{\mathrm{DP}}$ values increase slowly; while in the convective regions where rainfall rate may be higher, the $Z_{\mathrm{DR}}$ values are larger and the $\Phi_{\mathrm{DP}}$ values increase faster. The PIA optimized by the variational analysis from the radar site to each range gate is shown in Fig. 5d. Due to the generally higher $A_{H}$ associated with larger $K_{\mathrm{DP}}$, it can be found that the increase of PIA is larger in the heavy rainfall regions than the weak echo regions.

Figure 6 exhibits the further comparison of the $\alpha$ and total PIA based on different configurations of QPE for each ray of the scan in Fig. 5. To demonstrate the differences of DSD properties for different ray, we define a variable: the $K_{\mathrm{DP}}$-weighted $Z_{\mathrm{DR}}$ (denoted as $Z_{\mathrm{DR}}^{w}$ ), which is also shown in terms of azimuth in Fig. 6. The formula of $Z_{\mathrm{DR}}^{w}$ for an $n$-gate ray is

$$
Z_{\mathrm{DR}}^{w}=\frac{\sum_{i=1}^{n} Z_{\mathrm{DR}}(i) \times K_{\mathrm{DP}}(i)}{\sum_{i=1}^{n} K_{\mathrm{DP}}(i)},
$$

where $Z_{\mathrm{DR}}(i)$ and $K_{\mathrm{DP}}(i)$ are the $Z_{\mathrm{DR}}$ and $K_{\mathrm{DP}}$ at the $i$ th gate calculated from the optimized state vector of the variational approach. In the definition of $Z_{\mathrm{DR}}^{w}, Z_{\mathrm{DR}}$ is an indicator of mean raindrop size, which is a monotonically increasing function of $D_{m}$ if the gamma distribution with a fixed shape parameter is assumed; $K_{\mathrm{DP}}$ has a quasi-linear relation with rainfall rate (Bringi and Chandrasekar 2001). Thus, $Z_{\mathrm{DR}}^{w}$ can be used as a proxy for rainfall rate-weighted mean raindrop size, similar to that defined in Ryzhkov et al. (2005a). In Fig. 6a, it is found that the ray-averaged $\alpha$ values from the variational approach for the VAZ (the green solid line) show large azimuthal variances. The maximum value $(\sim 0.47 \mathrm{~dB}$ per degree) is approximately twice the minimum value ( $\sim 0.23 \mathrm{~dB}$ per degree). Despite the impact of measurement errors, most of the 

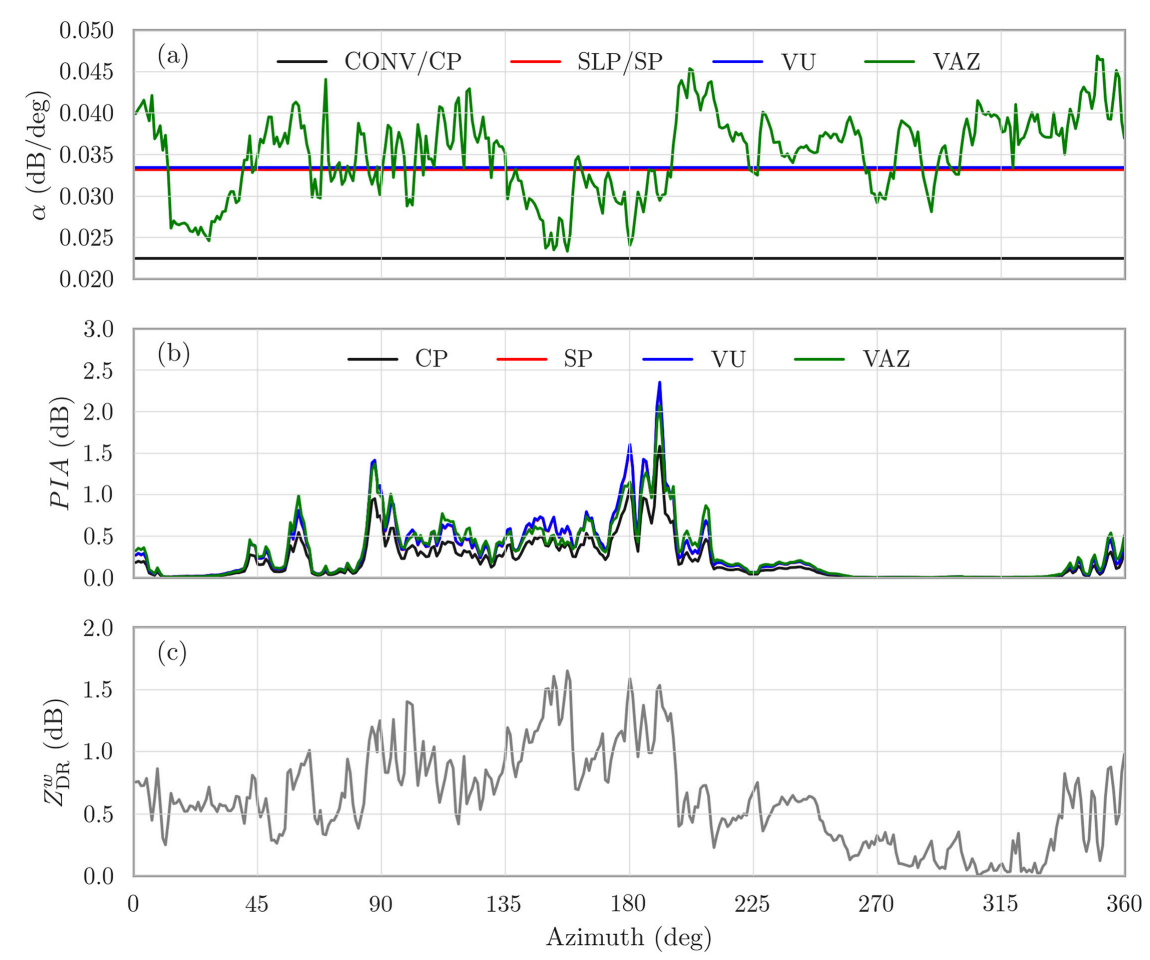

FIG. 6. (a) The $\alpha$ used for the CONV/CP, SLP/SP, VU, and VAZ and (b) the corresponding max PIA as functions of azimuth angle corresponding to the radar scan in Fig. 5. (c) The $Z_{\mathrm{DR}}^{w}$ for each ray is also shown.

variances are caused by the differences in the DSD properties of different rays. The ray-averaged $\alpha$ values show clear relations with the $Z_{\mathrm{DR}}^{w}$; the $\alpha$ values for the VAZ generally decreases when the $Z_{\mathrm{DR}}^{w}$ increases in the azimuth. This is consistent with decreasing in $\alpha$ with the increase of $D_{m}$ in Fig. 2, which may indicate that the impact of azimuthal DSD variabilities can be accounted for by the ray-averaged $\alpha$ values from the variational approach. For the azimuthal angles between $135^{\circ}$ and $190^{\circ}$ where most convective lines with larger $Z_{\mathrm{DR}}$ exist, it can be found that the ray-averaged $\alpha$ values are generally low compared to the other regions. For the CONV, CP, SLP, $\mathrm{SP}$, and $\mathrm{VU}$, constant $\alpha$ values are used for different azimuths in the PIA calculation. The $\alpha$ determined from the $Z_{\mathrm{DR}}$ slope of the radar sweep for the SLP and SP (red line) is about $0.033 \mathrm{~dB}$ per degree, and it is close to the sweep-averaged value from the variational approach for the VU (blue line). The $\alpha$ determined from DSD statistics for the CONV and CP (black line) is generally too low compared to the optimized values from the other two methods.

In Fig. 6b, due to the differences in the $\alpha$ values, the maximum PIA values for different QPE configurations are not the same. The PIA values from the SP and VU are close because of the close $\alpha$ values. For the azimuthal angles where most convective lines with larger
$Z_{\mathrm{DR}}$ exist $\left(135^{\circ}-190^{\circ}\right)$, the PIA values estimated for the VAZ are generally smaller than the ones for the SP and VU. This may indicate the potential overestimation of PIA and the rainfall rate in the convective regions, which is also pointed out by Wang et al. (2019).

\section{b. QPE evaluation in seven cases}

The comparison of radar-derived hourly rainfall against the gauge measurements in seven cases in May-June 2016 is shown in Table 2. The performance of the attenuationbased QPE approaches varies in different cases. With no DSD uncertainty considered, the performance of the CONV is generally the worst, with generally the lowest CC, highest RMSE, highest NE, and highest RB in each case. For the SLP, the $\alpha$ values are optimized by $Z_{\mathrm{DR}}$ slopes for radar sweeps, and the SLP results in better rainfall estimates than the CONV. Compared to the CONV and SLP, the $\Delta \Phi_{\mathrm{DP}}$ obtained from the forward operator of the variational approach is used for the calculation of PIA in (5) by the CP and SP. As a result, increases in CC values and decreases in RMSE values can be found for the results of the SP (CP) compared to those for the SLP (CONV). Similar to the SLP and SP, the $\alpha$ values are optimized for each sweep for the VU with the variational approach applied. As a result, the performance of the VU is also close to the SP. The accuracy of 
TABLE 2. The comparisons of the hourly rainfall estimated based on four configurations and those derived from the rain gauges in seven precipitation events in May-June 2016, including the correlation coefficient (CC), root-mean-squared error (RMSE), normalized error (NE), and relative bias (RB).

\begin{tabular}{|c|c|c|c|c|c|c|c|c|c|c|c|c|}
\hline & CONV & SLP & $\mathrm{CP}$ & SP & VU & VAZ & CONV & SLP & $\mathrm{CP}$ & SP & VU & VAZ \\
\hline Time & \multicolumn{6}{|c|}{0000 UTC 9 May-1200 UTC 10 May } & \multicolumn{6}{|c|}{1700 UTC 19 May-0300 UTC 21 May } \\
\hline $\mathrm{CC}$ & 0.92 & 0.92 & 0.93 & 0.93 & 0.93 & 0.94 & 0.88 & 0.89 & 0.90 & 0.91 & 0.91 & 0.92 \\
\hline $\operatorname{RMSE}(\mathrm{mm})$ & 3.70 & 3.63 & 3.57 & 3.51 & 3.50 & 3.17 & 2.49 & 2.39 & 2.28 & 2.16 & 2.04 & 1.99 \\
\hline $\mathrm{NE}$ & 0.38 & 0.38 & 0.35 & 0.35 & 0.35 & 0.33 & 0.52 & 0.51 & 0.44 & 0.43 & 0.41 & 0.41 \\
\hline $\mathrm{RB}(\%)$ & 3.1 & 3.4 & 1.0 & 1.2 & 4.2 & 2.3 & -31.0 & -25.6 & -27.4 & 21.8 & -11.5 & -12.6 \\
\hline Time & \multicolumn{6}{|c|}{0000 UTC 27 May-1200 UTC 28 May } & \multicolumn{6}{|c|}{0300 UTC 4 Jun-1100 UTC 4 Jun } \\
\hline $\mathrm{CC}$ & 0.92 & 0.92 & 0.94 & 0.93 & 0.94 & 0.94 & 0.91 & 0.91 & 0.91 & 0.92 & 0.91 & 0.91 \\
\hline $\operatorname{RMSE}(\mathrm{mm})$ & 1.88 & 1.81 & 1.77 & 1.65 & 1.63 & 1.54 & 3.73 & 3.64 & 3.70 & 3.62 & 3.57 & 3.38 \\
\hline $\mathrm{NE}$ & 0.48 & 0.49 & 0.42 & 0.41 & 0.41 & 0.40 & 0.43 & 0.43 & 0.44 & 0.45 & 0.48 & 0.46 \\
\hline $\mathrm{RB}(\%)$ & -21.5 & 2.8 & -22.8 & -0.1 & 2.6 & 0.5 & 23.4 & 23.3 & 27.3 & 27.7 & 28.7 & 27.3 \\
\hline Time & \multicolumn{6}{|c|}{1800 UTC 4 Jun-1300 UTC 5 Jun } & \multicolumn{6}{|c|}{0400 UTC 7 Jun-0900 UTC 7 Jun } \\
\hline $\mathrm{CC}$ & 0.90 & 0.91 & 0.91 & 0.91 & 0.91 & 0.92 & 0.85 & 0.84 & 0.85 & 0.84 & 0.84 & 0.85 \\
\hline $\operatorname{RMSE}(\mathrm{mm})$ & 2.05 & 2.11 & 2.00 & 2.09 & 2.06 & 1.93 & 4.07 & 4.19 & 4.02 & 4.18 & 4.53 & 3.79 \\
\hline $\mathrm{NE}$ & 0.35 & 0.36 & 0.32 & 0.34 & 0.34 & 0.33 & 0.63 & 0.63 & 0.60 & 0.60 & 0.64 & 0.59 \\
\hline $\mathrm{RB}(\%)$ & -8.9 & -14.6 & -13.3 & -18.6 & 5.7 & 3.9 & 28.7 & 26.8 & 27.4 & 25.8 & 31.3 & 26.1 \\
\hline Time & \multicolumn{6}{|c|}{2000 UTC 27 Jun-1100 UTC 28 Jun } & & & & & & \\
\hline $\mathrm{CC}$ & 0.85 & 0.86 & 0.87 & 0.87 & 0.87 & 0.89 & & & & & & \\
\hline $\mathrm{RMSE}(\mathrm{mm})$ & 3.39 & 3.44 & 3.23 & 3.26 & 3.35 & 2.88 & & & & & & \\
\hline $\mathrm{NE}$ & 0.45 & 0.42 & 0.41 & 0.38 & 0.38 & 0.35 & & & & & & \\
\hline $\mathrm{RB}(\%)$ & -23.9 & -11.2 & -21.5 & -8.2 & -5.0 & -6.4 & & & & & & \\
\hline
\end{tabular}

the VAZ is the best among the six approaches, with the highest CC, the lowest RMSE, and the lowest NE, while the $\mathrm{RB}$ values for the VAZ are close to those for the VU. The good performance of the VAZ may be attributed to the consideration of azimuthal DSD variabilities in the ray-averaged $\alpha$.

Since the performance of the SP is similar to that of the VU, we compare the $\alpha$ values for the two configurations in Fig. 7. These two configurations aim to considered the DSD properties in the optimization of $\alpha$ by using the $\alpha-K$ relation (the SP) and the variational analysis (the UV). It can be found that the general trends of the $\alpha$ derived from the $Z_{\mathrm{DR}}$ slope and the sweep-averaged $\alpha$ from the variational analysis are similar. For example, in the precipitation event between 0000 UTC 27 May and 1200 UTC 28 May, the $\alpha$ values for the SP and UV are very close. But differences still exist in the sweep-averaged $\alpha$ values from these two configurations. This is mainly because of their different

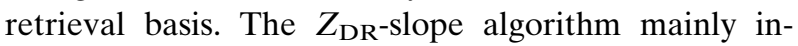
volves an assumption of the relation between the mean raindrop size and the $Z_{\mathrm{DR}}$ slope $K$, while the variational approach uses the relations among polarimetric variables which has accounted for most DSD variabilities. Besides, in the $\alpha$ estimation from $Z_{\mathrm{DR}}$ slope, the $\alpha$ estimates only update when enough valid samples exist or accumulate, which results in the steps in the corresponding $\alpha$ curves. This strategy mitigates the impact of measurement errors while gains the impact of DSD variabilities for the sweeps without enough samples for $\alpha$ estimation. On the other hand, one sweepaveraged $\alpha$ value is determined for one radar sweep in the variational method. For the radar sweep with a small sample size, larger $\alpha$ uncertainty may be caused by the measurement errors. This could be the reason for the larger sweep-to-sweep $\alpha$ variabilities between 0000 and 1200 UTC 9 May. However, considering the performance of the UV, this uncertainty caused by measurement errors is acceptable for the accuracy of QPE and is not further processed here. In Fig. 7, the $\alpha$ values (about $0.023 \mathrm{~dB}$ per degree in all the cases) from DSD statistics are also shown for comparison, which results in the relatively worse performance of CONV and CP.

The performance of the VAZ is the best compared to the other configurations denoted by Table 2. This is mainly because the $\alpha$ value is optimized for each radar ray for the VAZ, in which the DSD properties, e.g., smaller or larger mean raindrop size, in the radar ray are considered, which has been revealed by Fig. 6. To further illustrate this, it is useful to show the azimuthal and temporal variabilities in the optimized $\alpha$ values and the $Z_{\mathrm{DR}}^{w}$, which is an indicator of the mean raindrop size for rays. The result for the precipitation event over the period 0000 UTC 27 May-1200 UTC 28 May 2016 is shown in Fig. 8. Besides the temporal variabilities which have been shown by the sweep-averaged $\alpha$ values used by the 

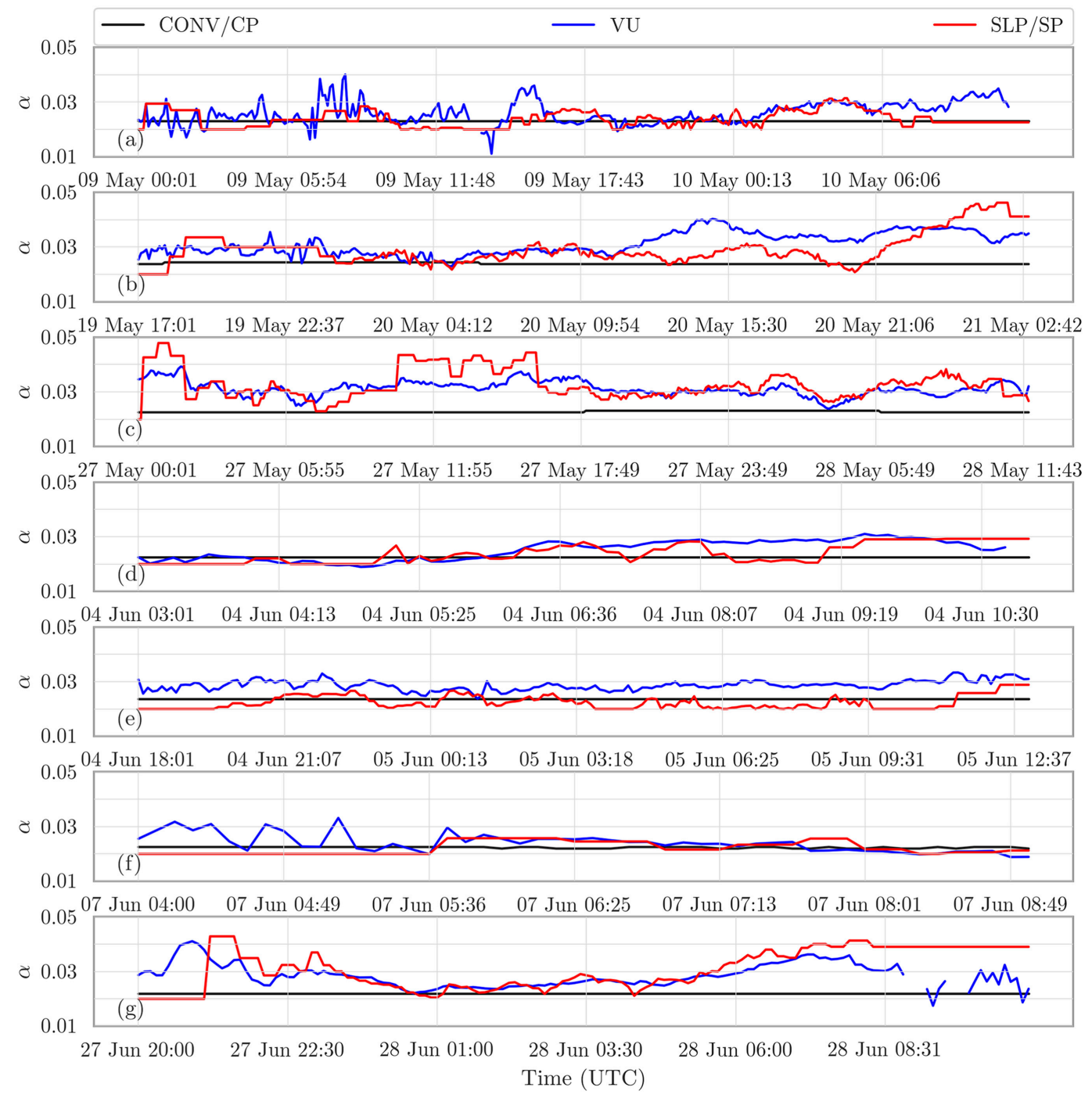

FIG. 7. The time series of sweep-averaged $\alpha$ values (dB per degree) (blue solid lines) obtained by the variational approach (i.e., the ones used in the UV) for the precipitation events shown in Table 2. The black (red) solid lines represent the $\alpha$ values used in the CP and CONV (SLP and SP).

VU (Fig. 7), the ray-optimized $\alpha$ values (Fig. 8a) also show clear azimuthal variabilities for the whole event. Comparing the $\alpha$ values optimized for the radar rays (Fig. 8a) with the $Z_{\mathrm{DR}}^{w}$ values (Fig. 8b), it can be found that the larger $\alpha$ values are generally corresponding to the lower $Z_{\mathrm{DR}}^{w}$, which indicates lower mean raindrop sizes. This is consistent with the change of $\alpha$ in terms of $D_{m}$ in Fig. 2. If a uniform $\alpha$ is used for a whole sweep, the rainfall rate tends to be overestimated (underestimated) for the rays with larger (smaller) mean raindrop sizes. By considering the azimuthal DSD variabilities (indicated by the values of $Z_{\mathrm{DR}}^{w}$ ) in the optimization of $\alpha$ values, the VAZ results more accurate rainfall estimation than the other configurations with higher CC, lower RMSE and lower NE values (Table 2).
To show the overall performance of rainfall estimation in May-June 2016, Fig. 9 exhibits the comparisons of radar-derived hourly rainfall using the above six QPE configurations against the gauge measurements in all the seven cases, in which the total sample size exceeds 40000 . Similar to the results shown in Table 2, the hourly rainfall estimated by the CONV (Fig. 9a) has the worst performance due to the utilization of fixed $\alpha$ values derived from the statistics of the DSD observations; clear underestimation $(\mathrm{RB}=-10.4 \%)$ can also be found along with the lowest CC (0.90), largest RMSE $(2.75 \mathrm{~mm})$, and largest NE (0.44). The results of the CP (Fig. 9c) are better due to the utilization of $\Delta \Phi_{\mathrm{DP}}$ from the variational analysis, but underestimation still exists. Compared to the CONV (Fig. 9a), the SLP (Fig. 9b) uses the $\alpha$ values optimized 

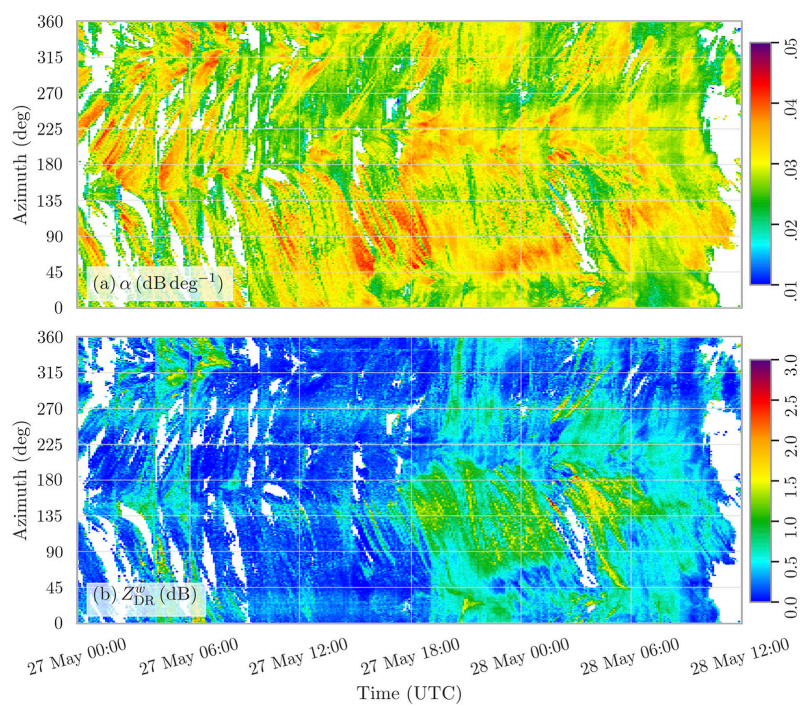

FIG. 8. The ray-averaged (a) $\alpha$ and(b) $Z_{\mathrm{DR}}^{w}$ for radar rays as functions of time and azimuth in the precipitation event occurred from 0000 UTC 27 May to 1200 UTC 28 May 2016.

by the $Z_{\mathrm{DR}}$ slope, which results in the slightly lower RMSE $(2.70 \mathrm{~m})$, NE (0.43), and RB (-4.5\%). The improvement over the results from the SLP (Fig. 9a) can also be found for those from the SP (Fig. 9d) for which the $\Delta \Phi_{\mathrm{DP}}$ has been optimized. This improvement is even larger than the improvement of the SLP over the CONV in our cases, especially according to the decreases of the RMSE and NE. For the VU which also uses sweepaveraged $\alpha$ values but from the variational analysis (Fig. 9e), the performance is similar to that of the SP (Fig. 9d), with similar CC, RMSE, and NE. In the VAZ, due to the consideration of azimuthal DSD variabilities (see Fig. 8a), the rainfall estimates have the highest CC (0.92), lowest RMSE $(2.35 \mathrm{~mm})$, lowest $\mathrm{NE}$, and lowest $\mathrm{RB}$ in the comparison against the gauge measurements, which proves that the VAZ is the best configuration for attenuation-based QPE.

At last, the verification of radar-derived total accumulated rainfalls against the gauge measurements in the cases in Table 2 is shown in Fig. 10. Since the CONV and SLP have worse performance for the estimation of hourly rainfalls than the other configurations according to Fig. 9, their results for the total accumulated rainfalls are not shown here. In Fig. 10, it is found that all the three configurations (SP, VU, and VAZ) with $\alpha$ values optimized can generally result in good total accumulated rainfalls in May-June 2016, except some rainfall underestimation far from the radar site. At these range gates farther away from the radar site, the sampling altitudes are higher. Due to the dominance of the collision-coalescence process, the rainfall rate of south China generally increases toward the ground (Liu and
Zipser 2013; Wu et al. 2018), which probably is the reason for the underestimation. Besides, the terrain is also higher in these regions far away from the radar site, which could cause larger uncertainty. The improvement of results based on the VAZ over those from the VU and the SP is not very significant, mainly because the impact of DSD uncertainty in different azimuths becomes lower in the long-term accumulation of rainfall. In contrast to the results of the SP, the VU, and the VAZ, the results based on the $\mathrm{CP}$ show more significant underestimation. This is because the $\alpha$ values only from DSD statistics are too low for these cases.

\section{Conclusions and discussion}

In consideration of the good relation between $R$ and $A_{H}$, the $A_{H}$ values estimated from the ZPHI algorithm are used for rainfall estimation for GZ SPOL in south China. A variational approach based on the relations among polarimetric variables is proposed to optimize the coefficient $\alpha$ in the ZPHI algorithm, which is compared against an approach for $\alpha$ optimization based on a slope of $Z_{\mathrm{DR}}$ dependence on $Z_{H}$. Six configurations for attenuation-based rainfall estimation are designed to show the performance of these approaches.

Generally, the hourly rainfall based on the CONV has the worst performance with a significant underestimation, mainly because the $\alpha$ values are derived from the climatic measurements of 2DVD without consideration of the DSD variabilities in specific cases. The SLP with $\alpha$ optimized based on the $Z_{\mathrm{DR}}$ slope for each radar sweep shows better performance (a lower system bias) than the CONV. Besides the accuracy of $\alpha$, the random errors in $\Phi_{\mathrm{DP}}$ also have an impact on the accuracy of the estimation of PIA, which further affects the accuracy of attenuation and rainfall estimation. Once the $\phi_{\mathrm{DP}}$ optimized by the variational approach is utilized for the calculation of the $\Delta \Phi_{\mathrm{DP}}$ in the CP (SP) configuration, the $\mathrm{CC}$ between the radar-derived hourly rainfall against the gauge measurements increases and the RMSE/NE decreases compared to the results of the CONV (SLP). However, the system bias generally has a minor change according to the RB values. Similar to the SP, sweepaveraged $\alpha$ values are derived from the variational approach, which is used for QPE in the VU. The VU has a similar performance to the SP for rainfall estimation $(\mathrm{CC}=0.92, \mathrm{RMSE}=2.56 \mathrm{~mm}$, and $\mathrm{NE}=0.39)$. The advantage of the variational approach is that an averaged $\alpha$ value can be calculated for each radar ray instead of the whole radar sweep. In such a way, the azimuthal DSD variability can be mitigated in the $\alpha$ optimization and the corresponding QPE. For the VAZ which uses the rayaveraged $\alpha$, the accuracy of the estimated hourly rainfall 

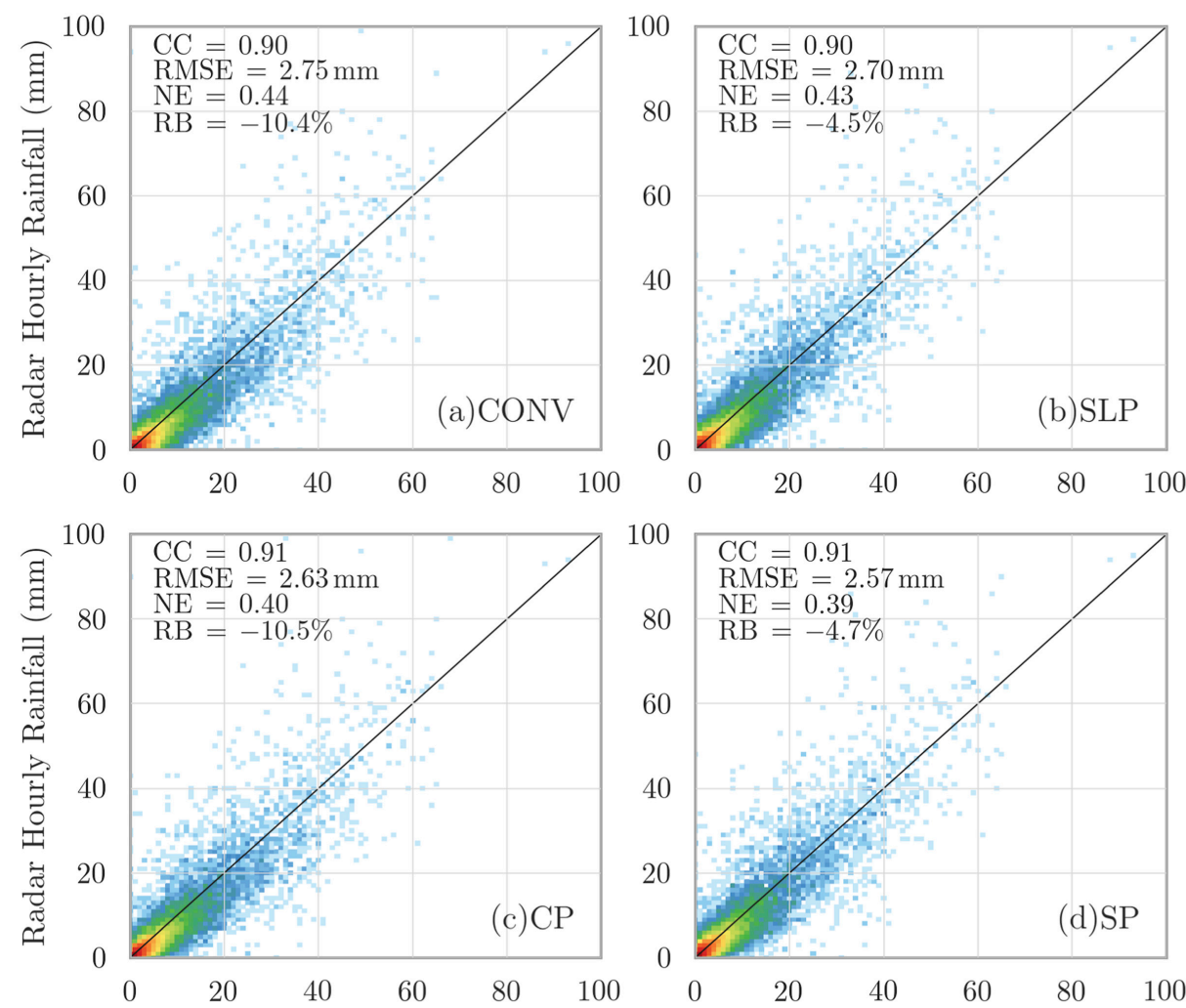

100
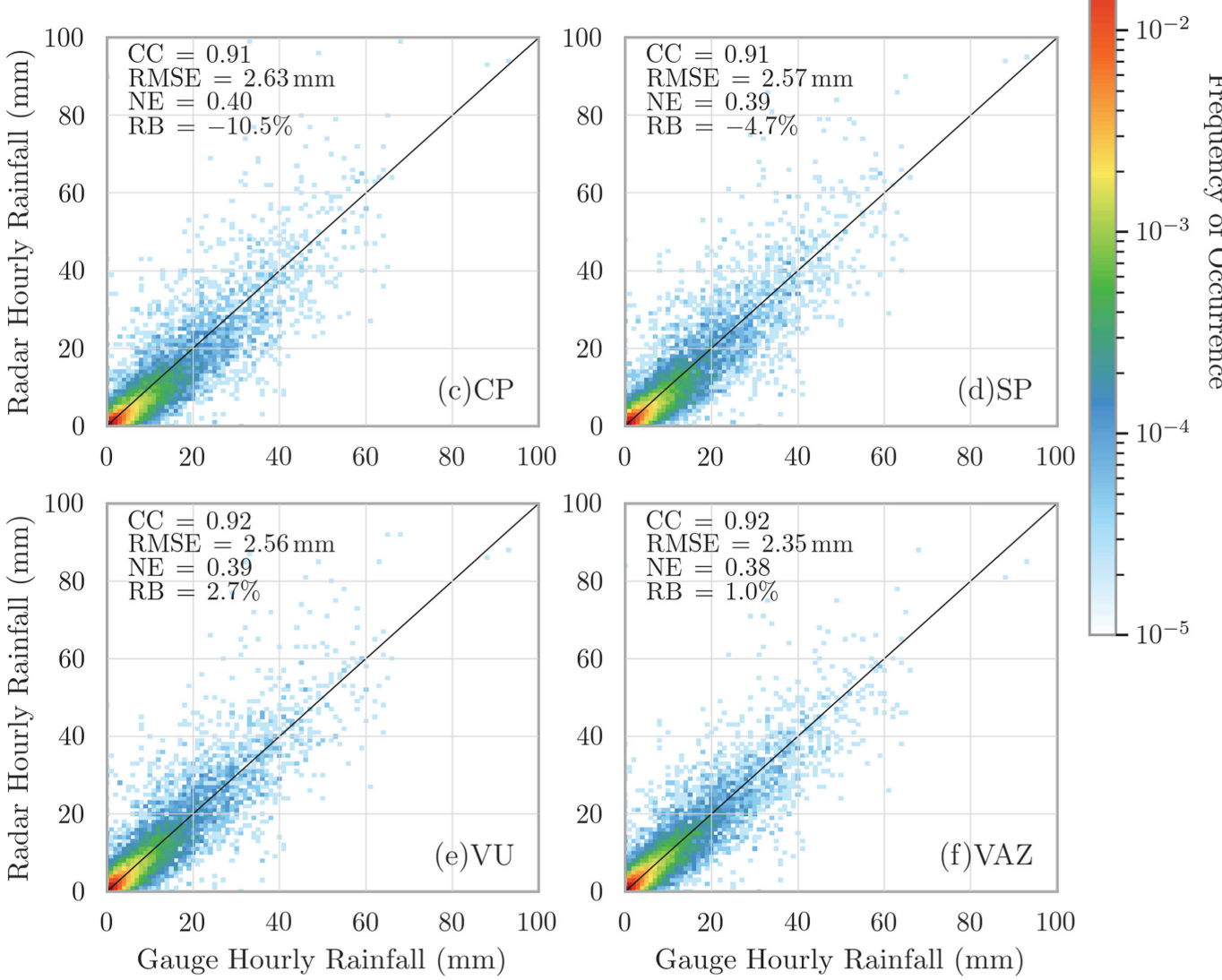

FIG. 9. The joint distributions (shading) of the hourly rainfall amounts from the gauge measurements vs those derived from the PPI scans of GZ SPOL at the elevation angle of $1.5^{\circ}$ for all seven cases listed in Table 2 . The category sizes of the hourly rainfall amounts are $1 \mathrm{~mm}$. (a)-(f) The results for the attenuation-based rainfall estimation using the six different configurations: CONV, SLP, CP, SP, VU, and VAZ, respectively.

is further improved than the VU and SP. The RMSE decreases about $8 \%$ of that for the VU (from 2.56 to $2.35 \mathrm{~mm}$ ). According to the above results, the VAZ proves to be the best configuration for attenuationbased QPE. The results from the VAZ can be also compared against those obtained by the variational approach based on the $R-K_{\mathrm{DP}}$ relation used in Huang et al. (2018). It is revealed that the results from the VAZ have better performance with larger $\mathrm{CC}$ and lower RMSE values, which is mainly because the $R-A_{\mathrm{H}}$ relation suffers less from the DSD variabilities.

Although the variational approach has a good performance in the optimization of $\alpha$ and the rainfall estimation shown by the previous comparison, there are also limitations. First, even the relations among the polarimetric variables in Fig. 4 are generally solid, some outliers still exist especially in the parameterization of $A_{H}$ (Fig. 4b). Besides, in an individual case, the DSD variabilities can still cause 


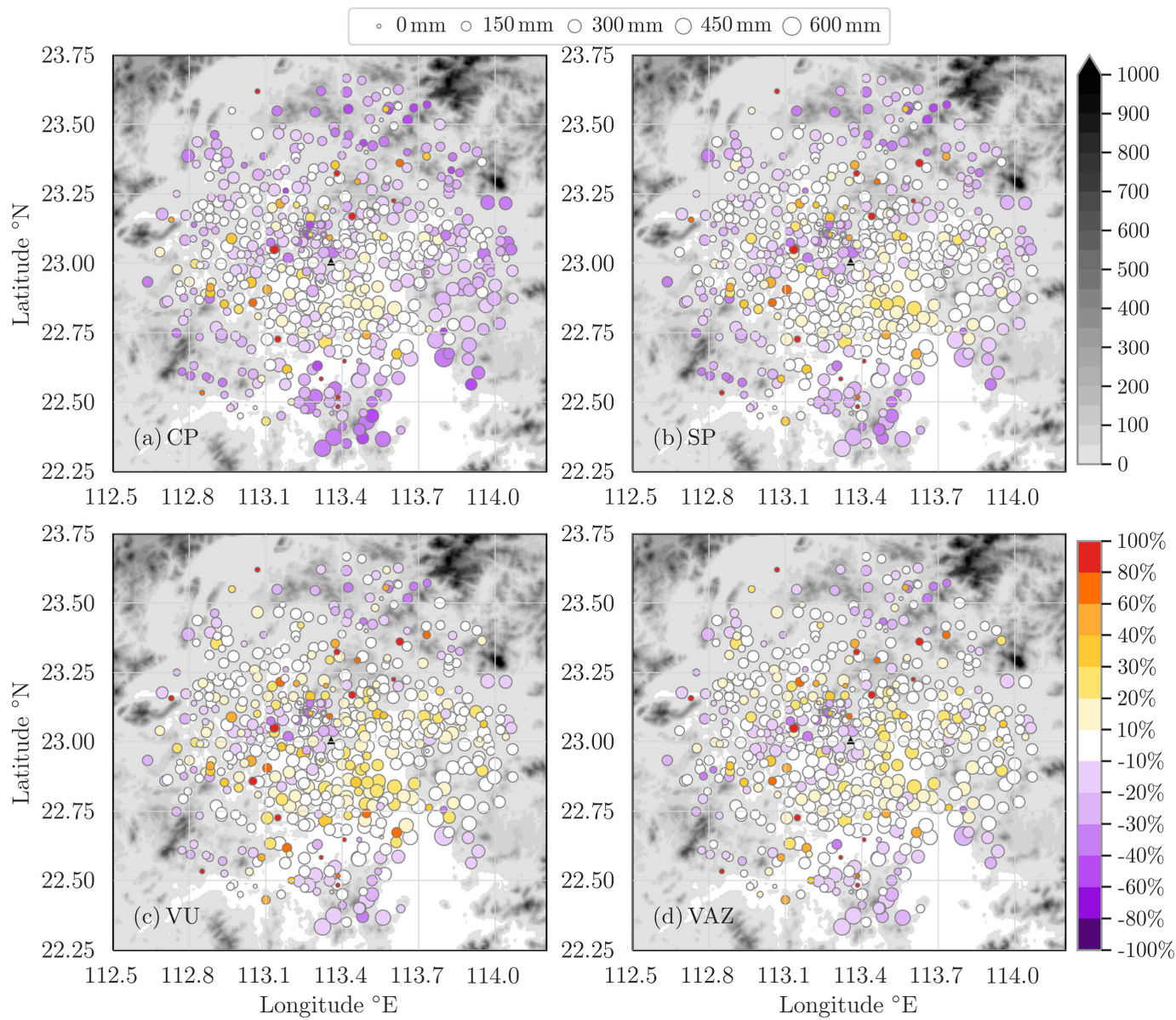

FIG. 10. Comparisons of the total accumulated rainfalls derived from the gauges and those from the PPI scans at the elevation angle of $1.5^{\circ}$ based on (a) the CP, (b) the SP, (c) the VU, and (d) the VAZ in all the cases listed in Table 2. The positions of the circles show the gauge locations. The size of the circles denotes the total accumulated rainfall from the rain gauges, and the color shading shows the relative bias of the radar-derived rainfalls. The gray shading represents the terrain height. The radar site position is marked as black triangles.

different levels of uncertainty in the forward operator based on the parameterization of $K_{\mathrm{DP}}, A_{H}$, and $A_{\mathrm{DP}}$ in terms of $Z_{H}$ and $Z_{\mathrm{DR}}$, which will further affect the QPE performance. This is probably why the QPE performance in different rainfall events (Table 2) varies.

Since the forward operator involves $Z_{H}$ and $Z_{\mathrm{DR}}$, the variational approach requires a good data quality of polarimetric variables. In our study, the quality control procedures are used to guarantee the accuracy of the radar variables for the variational analysis. However, currently, good data quality cannot always be expected from all operational radars due to the common calibration issues (Zittel et al. 2015), which may limit the applicability of the variational approach. As shown by Wang et al. (2019), the approach for $\alpha$ optimization based on the $Z_{\mathrm{DR}}$ slope is less affected by miscalibration, even though the azimuthal changes of the system biases of $Z_{H}$ and $Z_{\mathrm{DR}}$ can still impact its accuracy. Currently, the $\alpha$ optimization approach based on the $Z_{\mathrm{DR}}$ slope is more broadly applicable than the variational approach considering the data quality issues. However, the results of the VAZ demonstrate the potential room for improvement by considering the azimuthal variability of $\alpha$. It is also possible for the variational approach to segment the rainfall into finer subregions, for example, not only in azimuth but also in different ranges. However, in the meantime, a finer segmentation can reduce the sample size for $\alpha$ optimization and the impact of the radar measurement errors can increase. This topic is left for the future studies.

It is also worth mentioning that fixed standard deviations of the measurement errors of $Z_{H}(1 \mathrm{~dB}), Z_{\mathrm{DR}}$ $(0.2 \mathrm{~dB})$, and $\Phi_{\mathrm{DP}}\left(2^{\circ}\right)$ are used for the variational analysis according to the radar specification. This may not be realistic since the measurement errors of $Z_{H}$, $Z_{\mathrm{DR}}$, and $\Phi_{\mathrm{DP}}$ can increase when the signal-to-noise ratio or $\rho_{\mathrm{hv}}$ decreases. It is not easy to accurately account 
for this impact; however, this impact on retrieval is expected to be low because the measurement errors of $Z_{H}$, $Z_{\mathrm{DR}}$, and $\Phi_{\mathrm{DP}}$ are in the denominator terms of the cost function and can partially cancel with each other when they increase simultaneously.

Acknowledgments. The authors would also like to thank the scientists and engineers working on GZ SPOL, 2DVD, and the other instruments used in this study. These data used in this study can be accessed upon request but their usage may be restricted by relevant Chinese Government rules and regulations that are beyond the control of the authors. This work is primarily supported by the National Key Research and Development Program of China (Grant 2018YFC1506404 and Grant 2017YFC1501703) and the National Natural Science Foundation of China (Grant 41905019 and 41875053), the Open Research Program of the State Key Laboratory of Severe Weather, the Operational Technology Experiment and Demonstration Project for Hail Suppression and Precipitation Enhancement in the East Side of Taihang Mountain, China (HBRUWCSY_2017_04), the 5th "333 High-level Personnel Training Project" of Jiangsu Province (BRA2019037), the Guangzhou Science and Technology Project (201804020038), and the Science and Technology Project of Zhejiang Province (Grant 2017C03035).

\section{REFERENCES}

Brandes, E. A., G. Zhang, and J. Vivekanandan, 2002: Experiments in rainfall estimation with a polarimetric radar in a subtropical environment. J. Appl. Meteor., 41, 674-685, https://doi.org/ 10.1175/1520-0450(2002)041<0674:EIREWA > 2.0.CO;2.

Bringi, V. N., and V. Chandrasekar, 2001: Polarimetric Doppler Weather Radar: Principles and Applications. Cambridge University Press, 664 pp.

—, T. D. Keenan, and V. Chandrasekar, 2001: Correcting C-band radar reflectivity and differential reflectivity data for rain attenuation: A self-consistent method with constraints. IEEE Trans. Geosci. Remote Sens., 39, 1906-1915, https:// doi.org/10.1109/36.951081.

- M. A. Rico-Ramirez, and M. Thurai, 2011: Rainfall estimation with an operational polarimetric C-band radar in the United Kingdom: Comparison with a gauge network and error analysis. J. Hydrometeor., 12, 935-954, https://doi.org/10.1175/ JHM-D-10-05013.1.

Byrd, R. H., P. Lu, J. Nocedal, and C. Zhu, 1995: A limited memory algorithm for bound constrained optimization. SIAM J. Sci. Comput., 16, 1190-1208, https://doi.org/10.1137/0916069.

Cao, Q., M. B. Yeary, and G. Zhang, 2012: Efficient ways to learn weather radar polarimetry. IEEE Trans. Educ., 55, 58-68, https://doi.org/10.1109/TE.2011.2118211.

, G. Zhang, and M. Xue, 2013: A variational approach for retrieving raindrop size distribution from polarimetric radar measurements in the presence of attenuation. J. Appl. Meteor. Climatol., 52, 169-185, https://doi.org/10.1175/JAMC-D-12-0101.1.
Carey, L. D., S. A. Rutledge, D. A. Ahijevych, and T. D. Keenan, 2000: Correcting propagation effects in C-band polarimetric radar observations of tropical convection using differential propagation phase. J. Appl. Meteor., 39, 1405-1433, https://doi.org/ 10.1175/1520-0450(2000)039<1405:CPEICB > 2.0.CO;2.

Chen, H., V. Chandrasekar, and R. Bechini, 2017: An improved dualpolarization radar rainfall algorithm (DROPS2.0): Application in NASA IFloodS field campaign. J. Hydrometeor., 18, 917937, https://doi.org/10.1175/JHM-D-16-0124.1.

Chen, X., K. Zhao, and M. Xue, 2014: Spatial and temporal characteristics of warm season convection over Pearl River Delta region, China, based on 3 years of operational radar data. J. Geophys. Res. Atmos., 119, 12 447-12 465, https://doi.org/10.1002/2014jd021965.

Cocks, S. B., and Coauthors, 2019: A prototype quantitative precipitation estimation algorithm for operational S-band polarimetric radar utilizing specific attenuation and specific differential phase. Part II: Performance verification and case study analysis. J. Hydrometeor., 20, 999-1014, https://doi.org/10.1175/ JHM-D-18-0070.1.

Diederich, M., A. Ryzhkov, C. Simmer, P. Zhang, and S. Trömel, 2015: Use of specific attenuation for rainfall measurement at X-band radar wavelengths. Part II: Rainfall estimates and comparison with rain gauges. J. Hydrometeor., 16, 503-516, https://doi.org/10.1175/JHM-D-14-0067.1.

Gorgucci, E., G. Scarchilli, and V. Chandrasekar, 1992: Calibration of radars using polarimetric techniques. IEEE Trans. Geosci. Remote Sens., 30, 853-858, https://doi.org/10.1109/36.175319.

Gou, Y., H. Chen, and J. Zheng, 2019: An improved self-consistent approach to attenuation correction for C-band polarimetric radar measurements and its impact on quantitative precipitation estimation. Atmos. Res., 226, 32-48, https://doi.org/10.1016/ j.atmosres.2019.03.006.

Gu, J. Y., A. Ryzhkov, P. Zhang, P. Neilley, M. Knight, B. Wolf, and D. I. Lee, 2011: Polarimetric attenuation correction in heavy rain at C band. J. Appl. Meteor. Climatol., 50, 39-58, https://doi.org/10.1175/2010JAMC2258.1.

Hogan, R. J., 2007: A variational scheme for retrieving rainfall rate and hail reflectivity fraction from polarization radar. J. Appl. Meteor. Climatol., 46, 1544-1564, https://doi.org/10.1175/JAM2550.1.

_ 2014: Fast reverse-mode automatic differentiation using expression templates in $\mathrm{C}++$. ACM Trans. Math. Software, 40, 26, https://doi.org/10.1145/2560359.

Huang, H., and Coauthors, 2018: Quantitative precipitation estimation with operational polarimetric radar measurements in southern China: A differential phase-based variational approach. J. Atmos. Oceanic Technol., 35, 1253-1271, https:// doi.org/10.1175/JTECH-D-17-0142.1.

—, K. Zhao, G. Zhang, D. Hu, and Z. Yang, 2020: Optimized raindrop size distribution retrieval and quantitative rainfall estimation from polarimetric radar. J. Hydrol., 580, 124248, https://doi.org/10.1016/j.jhydrol.2019.124248.

Lee, G. W., 2006: Sources of errors in rainfall measurements by polarimetric radar: Variability of drop size distributions, observational noise, and variation of relationships between $\mathrm{R}$ and polarimetric parameters. J. Atmos. Oceanic Technol., 23, 1005-1028, https://doi.org/10.1175/JTECH1899.1.

Liu, C., and E. J. Zipser, 2013: Why does radar reflectivity tend to increase downward toward the ocean surface, but decrease downward toward the land surface? J. Geophys. Res. Atmos., 118, 135-148, https://doi.org/10.1029/2012JD018134.

Luo, Y., and Coauthors, 2017: The Southern China Monsoon Rainfall Experiment (SCMREX). Bull. Amer. Meteor. Soc., 98, 999-1013, https://doi.org/10.1175/BAMS-D-15-00235.1. 
Maesaka, T., K. Iwanami, and M. Maki, 2012: Non-negative KDP estimation by monotone increasing ФDP assumption below melting layer. Extended Abstracts, Seventh European Conf. on Radar in Meteorology and Hydrology, Toulouse, France, Météo-France, 26 QPE, http://www.meteo.fr/cic/meetings/ 2012/ERAD/extended_abs/QPE_233_ext_abs.pdf.

Marshall, J. S., and W. M. K. Palmer, 1948: The distribution of raindrops with size. J. Meteor., 5, 165-166, https://doi.org/ 10.1175/1520-0469(1948)005<0165:TDORWS >2.0.CO;2.

Mishchenko, M. I., J. W. Hovenier, and L. D. Travis, 2000: Light Scattering by Nonspherical Particles. Academic Press, 721 pp.

Ryzhkov, A. V., and D. S. Zrnić, 2019: Radar Polarimetry for Weather Observations. Springer, $497 \mathrm{pp}$.

_ S. E. Giangrande, and T. J. Schuur, 2005a: Rainfall estimation with a polarimetric prototype of WSR-88D. J. Appl. Meteor., 44, 502-515, https://doi.org/10.1175/JAM2213.1.

, T. J. Schuur, D. W. Burgess, P. L. Heinselman, S. E. Giangrande, and D. S. Zrnić, 2005b: The joint polarization experiment: Polarimetric rainfall measurements and hydrometeor classification. Bull. Amer. Meteor. Soc., 86, 809-824, https://doi.org/10.1175/BAMS-86-6-809.

- M. Diederich, P. Zhang, and C. Simmer, 2014: Potential utilization of specific attenuation for rainfall estimation, mitigation of partial beam blockage, and radar networking. J. Atmos. Oceanic Technol., 31, 599-619, https://doi.org/10.1175/JTECH-D-13-00038.1.

Testud, J., E. Le Bouar, E. Obligis, and M. Ali-Mehenni, 2000: The rain profiling algorithm applied to polarimetric weather radar.
J. Atmos. Oceanic Technol., 17, 332-356, https://doi.org/ 10.1175/1520-0426(2000)017<0332:TRPAAT > 2.0.CO;2.

Wang, Y., P. Zhang, A. V. Ryzhkov, J. Zhang, and P.-L. Chang, 2014: Utilization of specific attenuation for tropical rainfall estimation in complex terrain. J. Hydrometeor., 15, 2250-2266, https://doi.org/10.1175/JHM-D-14-0003.1.

—, S. Cocks, L. Tang, A. Ryzhkov, P. Zhang, J. Zhang, and K. Howard, 2019: A prototype quantitative precipitation estimation algorithm for operational S-band polarimetric radar utilizing specific attenuation and specific differential phase. Part I: Algorithm description. J. Hydrometeor., 20, 985-997, https://doi.org/10.1175/JHM-D-18-0071.1.

Wu, D., and Coauthors, 2018: Kinematics and microphysics of convection in the outer rainband of Typhoon Nida (2016) revealed by polarimetric radar. Mon. Wea. Rev., 146, 21472159, https://doi.org/10.1175/MWR-D-17-0320.1.

Zhang, J., and Coauthors, 2016: Multi-Radar Multi-Sensor (MRMS) quantitative precipitation estimation: Initial operating capabilities. Bull. Amer. Meteor. Soc., 97, 621-638, https://doi.org/ 10.1175/BAMS-D-14-00174.1.

Zittel, W. D., R. R. Lee, L. M. Richardson, J. G. Cunningham, J. A. Schultz, R. L. Ice, and R. O. Center, 2015: Geographical and seasonal availability of light rain, dry snow and Bragg scatter to estimate WSR-88D ZDR system bias. 31st Conf. on Environmental Information Processing Technologies, Phoenix, AZ, Amer. Meteor. Soc., 11.2, https://ams.confex.com/ ams/95Annual/webprogram/Paper265374.html. 